\title{
APPROXIMATION OF GAUSSIANS BY SPHERICAL GAUSS-LAGUERRE BASIS IN THE WEIGHTED HILBERT SPACE*
}

\author{
NADIIA DEREVIANKO ${ }^{\dagger, \ddagger}$ AND JÜRGEN PRESTIN ${ }^{\S}$
}

\begin{abstract}
This paper is devoted to the study of approximation of Gaussian functions by their partial Fourier sums of degree $N \in \mathbb{N}$ with respect to the spherical Gauss-Laguerre (SGL) basis in the weighted Hilbert space $L_{2}\left(\mathbb{R}^{3}, \omega_{\lambda}\right)$, where $\omega_{\lambda}(|\boldsymbol{x}|)=\exp \left(-|\boldsymbol{x}|^{2} / \lambda\right), \lambda>0$. We investigate the behavior of the corresponding error of approximation with respect to the scale factor $\lambda$ and order of expansion $N$. As interim results we obtained formulas for the Fourier coefficients of Gaussians with respect to SGL basis in the space $L_{2}\left(\mathbb{R}^{3}, \omega_{\lambda}\right)$. Possible application of obtained results to the docking problem are described.
\end{abstract}

Key words. spherical harmonic, Laguerre polynomial, Gaussian, hypergeometric function, molecular docking

AMS subject classifications. 33C05, 33C45, 33C55, 42C10

1. Introduction. Our goal in this paper is to study the behavior of the error of approximation of Gaussians by their partial Fourier sums with respect to the spherical Gauss-Laguerre (or shorter SGL) basis in the weighted Hilbert space $L_{2}\left(\mathbb{R}^{3}, \omega_{\lambda}\right)$, where

$$
\omega_{\lambda}(|\boldsymbol{x}|):=\exp \left(-|\boldsymbol{x}|^{2} / \lambda\right), \lambda>0, \boldsymbol{x}=(x, y, z) \in \mathbb{R}^{3} \quad \text { and } \quad|\boldsymbol{x}|=\sqrt{x^{2}+y^{2}+z^{2}}
$$

Motivation of our research comes from a wide range of applications of Gaussian in life sciences, in particular, in molecular modeling; see Section 5. The investigation of the behavior of the Fourier coefficients of Gaussians with respect to SGL basis functions in the space $L_{2}\left(\mathbb{R}^{3}, \omega_{\lambda}\right)$ is of special interest to us.

We denote the SGL basis by $\mathcal{H}^{\lambda}:=\left\{H_{n l m}^{\lambda}: \mathbb{R}^{3} \rightarrow \mathbb{C}, n \in \mathbb{N},(l, m) \in \nabla n\right\}$, where the parameter $\lambda>0$ and $\nabla_{n}:=\left\{(l, m) \in \mathbb{Z}^{2}: l=0, \ldots, n-1,|m|=0, \ldots, l\right\}$; see Section 2 for details. For a function $f \in L_{2}\left(\mathbb{R}^{3}, \omega_{\lambda}\right)$, by $\mathcal{E}_{N}\left(f, \mathcal{H}^{\lambda}\right)$ we denote the error of approximation of this function by its partial Fourier sum of order $N \in \mathbb{N}$ with respect to the basis $\mathcal{H}^{\lambda}$ in the space $L_{2}\left(\mathbb{R}^{3}, \omega_{\lambda}\right)$, i.e.,

$$
\mathcal{E}_{N}\left(f, \mathcal{H}^{\lambda}\right):=\left\|f-\sum_{n=1}^{N}\left(\sum_{(l, m) \in \nabla n} \widehat{f}_{n l m}^{\lambda} H_{n l m}^{\lambda}\right)\right\|_{L_{2}\left(\mathbb{R}^{3}, \omega_{\lambda}\right)}
$$

where $\widehat{f}_{n l m}^{\lambda}$ are Fourier coefficients of the function $f$ with respect to the basis $\mathcal{H}^{\lambda}$.

Our aim in this paper is to investigate the behavior of the quantity $\mathcal{E}_{N}\left(g, \mathcal{H}^{\lambda}\right)$ for the Gaussians $g\left(\boldsymbol{x}-\boldsymbol{x}_{0}\right)=\exp \left(-B\left|\boldsymbol{x}-\boldsymbol{x}_{0}\right|^{2}\right)$, where $B>0$ is fixed and a point $\boldsymbol{x}_{0} \in \mathbb{R}^{3}$ is given, with respect to the parameters $N \in \mathbb{N}$ and $\lambda>0$.

Let us motivate our choice of basis. The SGL basis is constructed by using a separation-ofvariables approach from the spherical harmonics and Laguerre polynomials. In the spherical coordinates

$$
H_{n l m}^{\lambda}(r, \theta, \varphi):=R_{n l}^{\lambda}(r) Y_{l m}(\theta, \varphi),
$$

* Received November 11, 2019. Accepted January 23, 2020. Published online on May 26, 2020. Recommended by Daniel Potts.

${ }^{\dagger}$ Fakultät für Mathematik, Technische Universität Chemnitz, 09107 Chemnitz, Germany.

${ }^{\ddagger}$ Institute of Mathematics of NAS of Ukraine, Tereshchenkivska st. 3, 01024 Kyiv-4, Ukraine (nadiia.derevianko@math.tu-chemnitz.de).

§Institut für Mathematik, Universität zu Lübeck, Ratzeburger Allee 160, 23562 Lübeck, Germany (prestin@math.uni-luebeck.de). 


\section{ETNA}

Kent State University and

Johann Radon Institute (RICAM)

the radial part $R_{n l}^{\lambda}(r)$ is defined via Laguerre polynomials, $\lambda>0$ is a scale factor and the spherical part $Y_{l m}(\theta, \varphi)$ is the spherical harmonic.

By $\Delta_{0}$ we denote the Laplace-Beltrami operator, i.e., the spherical part of the Laplace operator, that in spherical coordinates is given by

$$
\Delta_{0}:=\frac{1}{\sin \theta} \frac{\partial}{\partial \theta}\left(\sin \theta \frac{\partial}{\partial \theta}\right)+\frac{1}{\sin ^{2}} \frac{\partial^{2}}{\partial \varphi^{2}} .
$$

The spherical harmonics $Y_{l m}$ are eigenfunctions of $\Delta_{0}$, i.e.,

$$
\Delta_{0} Y_{l m}(\theta, \varphi)=-l(l+1) Y_{l m}(\theta, \varphi) .
$$

It is also known that spherical harmonics constitute an orthonormal basis of the space $L_{2}\left(\mathbb{S}^{2}\right)$ of square-integrable functions on the unit sphere $\mathbb{S}^{2}$ and are orthonormal in the sense that (see [4, Chap. 1])

$$
\int_{0}^{2 \pi} \int_{0}^{\pi} Y_{l m}(\theta, \varphi) \overline{Y_{l^{\prime} m^{\prime}}(\theta, \varphi)} \sin \theta \mathrm{d} \theta \mathrm{d} \varphi=\delta_{l l^{\prime}} \delta_{m m^{\prime}} .
$$

Because of these properties of spherical harmonics they are important in many areas of science and are widely used as a shape descriptor; see, for example, [18]. On the other hand, spherical harmonics are suitable for the approximation of smooth functions defined on the unit sphere $\mathbb{S}^{2}$. Problems of linear and nonlinear approximation of Sobolev classes of smooth functions defined on $\mathbb{S}^{d-1}$, where $d$ is the dimension of the space $\mathbb{R}^{d}$, by aggregates constructed by using spherical harmonics in the space $L_{q}\left(\mathbb{S}^{d-1}\right), 1 \leq q \leq \infty$, are investigated by many authors, for instance, by Kamzolov [10], Romanyuk [17], Dai and Xu [4], and Atkinson and Han [3]. From the viewpoint on practical applications in this paper we consider the case $d=3$.

In order to obtain full sampling of the space $\mathbb{R}^{3}$, we use a radial function that extends spherical harmonics to polynomials in this space. Different choices of radial functions are possible. As Ritchie and Kemp [16] (see also [14]), we employ the radial part constructed by using associated Laguerre polynomials $L_{k}^{\alpha}, k=0,1, \ldots$, and $\alpha>0$, that can be defined by the Rodrigues formula [7, p. 1051]

$$
L_{k}^{\alpha}(t):=\frac{t^{-\alpha} \exp (t)}{k !} \frac{\mathrm{d}^{k}\left(\exp (-t) t^{k+\alpha}\right)}{\mathrm{d} t^{k}}, t \in \mathbb{R} .
$$

Other radial functions such as Zernike polynomials may also be used; see [12] and [13] for details. Similarly to the angular zeros of the spherical harmonics, the Laguerre polynomials exhibit radial zeros and they are orthogonal with respect to a weight factor $\exp (-t) t^{\alpha}$, i.e.,

$$
\int_{0}^{\infty} \exp (-t) t^{\alpha} L_{k}^{(\alpha)}(t) L_{k^{\prime}}^{(\alpha)}(t) \mathrm{d} t=\frac{\Gamma(k+\alpha+1)}{k !} \delta_{k k^{\prime}} .
$$

In more general frameworks this basis is described in [5, Section 5.1.2].

Let us sketch the main results of the paper. The basic ingredient to investigate the behavior of the quantity $\mathcal{E}_{N}\left(g, \mathcal{H}^{\lambda}\right)$ is to use Parseval's equality and representation formulas for the SGL Fourier coefficients that we also obtain in this paper; see Section 3 and 4 for details. 


\section{ETNA}

Kent State University and

Johann Radon Institute (RICAM)

At first we consider the function $g\left(\boldsymbol{x}-\boldsymbol{x}_{0}\right)=\exp \left(-B\left|\boldsymbol{x}-\boldsymbol{x}_{0}\right|^{2}\right)$ in the case where $\boldsymbol{x}_{0}=\mathbf{0}$. For the quantity $\mathcal{E}_{N}\left(g, \mathcal{H}^{\lambda}\right)$, we have that

$$
\begin{aligned}
\mathcal{E}_{N}\left(g, \mathcal{H}^{\lambda}\right)= & \sqrt{\frac{2 \pi \Gamma\left(N+\frac{3}{2}\right)}{\Gamma(N+1)} \frac{\lambda^{\frac{3}{4}}}{(1+B \lambda)^{\frac{3}{2}}}\left(\frac{B \lambda}{1+B \lambda}\right)^{N}} \\
& \times \sqrt{{ }_{2} F_{1}\left(1, N+\frac{3}{2}, N+1 ;\left(\frac{B \lambda}{1+B \lambda}\right)^{2}\right)},
\end{aligned}
$$

where $\Gamma$ is the gamma function and ${ }_{2} F_{1}$ is the hypergeometric function; see the end of this section for a definition.

For $N \rightarrow \infty$ the asymptotic behavior of this quantity is described by (see Figure 3.1, left)

$$
\mathcal{E}_{N}\left(g, \mathcal{H}^{\lambda}\right) \sim C(\lambda, B)(N+1)^{\frac{1}{4}}\left(\frac{B \lambda}{1+B \lambda}\right)^{N},
$$

where the constant $C(\lambda, B)$ can be estimated as follows

$$
C(\lambda, B)=\frac{\sqrt{2 \pi} \lambda^{\frac{3}{4}}}{(1+B \lambda)^{\frac{3}{2}}} c(\lambda, B)
$$

and

$$
\frac{1+B \lambda}{\sqrt{1+2 B \lambda}}<c(\lambda, B) \leq\left(\frac{2}{3} \frac{(1+B \lambda)^{3}-(1+2 B \lambda)^{\frac{3}{2}}}{(B \lambda)^{2} \sqrt{1+2 B \lambda}}\right)^{\frac{1}{2}} \frac{1+B \lambda}{\sqrt{1+2 B \lambda}} .
$$

With respect to the parameter $\lambda$, the quantity $\mathcal{E}_{N}\left(g, \mathcal{H}^{\lambda}\right)$ is increasing on the segment $(0,+\infty)$ for all fixed $N \in \mathbb{N}$. Moreover, for all $N \in \mathbb{N}$ (see Figure 3.1, right)

$$
\lim _{\lambda \rightarrow \infty} \mathcal{E}_{N}\left(g, \mathcal{H}^{\lambda}\right)=\left(\frac{\pi}{2 B}\right)^{\frac{3}{4}} .
$$

For the function $g\left(\boldsymbol{x}-\boldsymbol{x}_{0}\right)=\exp \left(-B\left|\boldsymbol{x}-\boldsymbol{x}_{0}\right|^{2}\right)$ where $\boldsymbol{x}_{0} \neq 0$, we have numerical confirmation of the fact that the quantity $\mathcal{E}_{N}\left(g, \mathcal{H}^{\lambda}\right)$ keeps its behavior with respect to the parameter $\lambda>0$ when the point $\boldsymbol{x}_{0}$ is shifted from the origin. Unfortunately, due to the complex form of the SGL Fourier coefficients of Gaussians $g\left(\boldsymbol{x}-\boldsymbol{x}_{0}\right)$ in case $\boldsymbol{x}_{0} \neq 0$ it was not possible to get an analytical proof of this effect; see Section 4 for details. Note that with respect to $N \in \mathbb{N}$ it is easily seen from Parseval's equality that $\mathcal{E}_{N}\left(g, \mathcal{H}^{\lambda}\right)$ decays with respect to $N$, i.e., $\mathcal{E}_{N}\left(g, \mathcal{H}^{\lambda}\right) \geq \mathcal{E}_{N+1}\left(g, \mathcal{H}^{\lambda}\right)$ for all $N \in \mathbb{N}$.

The present paper has the following structure. In Section 2 we give the main definitions that are used in the paper. Section 3 contains a proof of the relations (1.1), (1.2) and (1.3) as well as formulas for the spherical Gauss-Laguerre Fourier coefficients for the function $g(\boldsymbol{x})=\exp \left(-B|\boldsymbol{x}|^{2}\right)$. In Section 4 we obtain formulas for the SGL Fourier coefficients of the functions $g\left(\boldsymbol{x}-\boldsymbol{x}_{0}\right)=\exp \left(-B\left|\boldsymbol{x}-\boldsymbol{x}_{0}\right|^{2}\right)$ in case when $\boldsymbol{x}_{0} \neq 0$ and show numerical results with respect to the behavior of the quantity $\mathcal{E}_{N}\left(g, \mathcal{H}^{\lambda}\right)$ for these functions. In Section 5 we describe one possible application of the obtained results to the docking problem.

Notation. As usual $\mathbb{N}=\{1,2,3, \ldots\}, \mathbb{R}$, and $\mathbb{C}$ denote the natural, real, and complex numbers, respectively. Consequently, $\mathbb{R}^{3}$ is the set of all vectors $\boldsymbol{x}=(x, y, z)$ and $|\boldsymbol{x}|=\sqrt{x^{2}+y^{2}+z^{2}}$ is the Euclidean norm of a vector $\boldsymbol{x}$. Letters $C_{i}, i=1,2, \ldots$, denote positive constants. We indicate in the brackets dependency on some parameters. By $\Gamma$ we 
denote the gamma function. Let $(t)_{n}=\frac{\Gamma(t+n)}{\Gamma(t)}, n \in \mathbb{N}$, and $(t)_{0}=1$ be the Pochhammer symbol [1, p. 256]. By ${ }_{2} F_{1}$ we denote the hypergeometric function $[7, \S 9.1]$

$$
{ }_{2} F_{1}(a, b, c, t)=\sum_{n=0}^{\infty} \frac{(a)_{n}(b)_{n}}{(c)_{n}} \frac{t^{n}}{n !},|t|<1
$$

Further, for given functions $f$ and $h$ we define a binary relation $f(t) \sim h(t)$ as $t \rightarrow \infty$ if and only if $\lim _{t \rightarrow \infty} \frac{f(t)}{h(t)}=1$. The symbol $\delta_{i j}$ is the Kronecker delta and as usual

$$
\delta_{i j}= \begin{cases}0 & \text { if } i \neq j \\ 1 & \text { if } i=j\end{cases}
$$

2. Preliminaries. As stated in the introduction the main goal of this paper is to investigate the behavior of the error of approximations of Gaussians by their partial Fourier sums with respect to the spherical Gauss-Laguerre (or SGL) basis in the weighted Hilbert space $L_{2}\left(\mathbb{R}^{3}, \omega_{\lambda}\right)$. In this section we give the definition of this basis.

By $L_{2}\left(\mathbb{R}^{3}, \omega_{\lambda}\right)$, where $\omega_{\lambda}(|\boldsymbol{x}|)=\exp \left(-|\boldsymbol{x}|^{2} / \lambda\right), \boldsymbol{x} \in \mathbb{R}^{3}$ and $\lambda>0$, we denote the weighted Hilbert space

$$
L_{2}\left(\mathbb{R}^{3}, \omega_{\lambda}\right):=\left\{f: \mathbb{R}^{3} \rightarrow \mathbb{C}, \int_{\mathbb{R}^{3}}|f(\boldsymbol{x})|^{2} \omega_{\lambda}(|\boldsymbol{x}|) \mathrm{d} \boldsymbol{x}<\infty\right\} .
$$

The inner product in this space is defined as

$$
\langle f, h\rangle_{L_{2}\left(\mathbb{R}^{3}, \omega_{\lambda}\right)}:=\int_{\mathbb{R}^{3}} f(\boldsymbol{x}) \overline{h(\boldsymbol{x})} \omega_{\lambda}(|\boldsymbol{x}|) \mathrm{d} \boldsymbol{x}, f, h \in L_{2}\left(\mathbb{R}^{3}, \omega_{\lambda}\right)
$$

and the norm as

$$
\|f\|_{L_{2}\left(\mathbb{R}^{3}, \omega_{\lambda}\right)}:=\sqrt{\langle f, f\rangle_{L_{2}\left(\mathbb{R}^{3}, \omega_{\lambda}\right)}}, f \in L_{2}\left(\mathbb{R}^{3}, \omega_{\lambda}\right) .
$$

Further, we define the SGL basis functions. To this end we use the spherical coordinates $(r, \theta, \varphi)$, where $r \in[0, \infty)$ is a radius, $\theta \in[0, \pi]$ is a polar angle, $\varphi \in[0,2 \pi)$ is an azimuthal angle, to write the Cartesian coordinates $x, y$, and $z$ as

$$
\begin{aligned}
& x=r \sin \theta \cos \varphi, \\
& y=r \sin \theta \sin \varphi, \\
& z=r \cos \theta .
\end{aligned}
$$

In the following we write $f(\boldsymbol{x})=f(r, \theta, \varphi)$ if $(r, \theta, \varphi)$ are spherical coordinates of the point $\boldsymbol{x}=(x, y, z)$, in which case we simply write $\boldsymbol{x}=(r, \theta, \varphi)$.

Then the inner product (2.1) can be rewritten as

$$
\langle f, h\rangle_{L_{2}\left(\mathbb{R}^{3}, \omega_{\lambda}\right)}:=\int_{0}^{\infty} \int_{0}^{\pi} \int_{0}^{2 \pi} f(r, \theta, \varphi) \overline{h(r, \theta, \varphi)} \omega_{\lambda}(r) r^{2} \sin \theta \mathrm{d} \varphi \mathrm{d} \theta \mathrm{d} r, f, h \in L_{2}\left(\mathbb{R}^{3}, \omega_{\lambda}\right),
$$

where $r^{2} \sin \theta$ is the Jacobian of the transform from Cartesian to spherical coordinates. 
Let us recall that $\nabla_{n}$ denotes the following set of indices

$$
\nabla_{n}:=\left\{(l, m) \in \mathbb{Z}^{2}: l=0, \ldots, n-1,|m|=0, \ldots, l\right\} .
$$

For $\lambda>0$ by $\mathcal{H}^{\lambda}=\left\{H_{n l m}^{\lambda}: \mathbb{R}^{3} \rightarrow \mathbb{C}, n \in \mathbb{N},(l, m) \in \nabla_{n}\right\}$ we denote the system of functions

$$
H_{n l m}^{\lambda}(r, \theta, \varphi):=R_{n l}^{\lambda}(r) Y_{l m}(\theta, \varphi),
$$

where the radial part $R_{n l}^{\lambda}$ is defined as

$$
R_{n l}^{\lambda}(r)=\left[\frac{2(n-l-1) !}{\lambda^{\frac{3}{2}} \Gamma\left(n+\frac{1}{2}\right)}\right]^{\frac{1}{2}}(r / \sqrt{\lambda})^{l} L_{n-l-1}^{\left(l+\frac{1}{2}\right)}\left(r^{2} / \lambda\right),
$$

where the $L_{k}^{\alpha}$ are associated Laguerre polynomials and $\lambda>0$ is a scale factor. The spherical part $Y_{l m}$ is represented by spherical harmonics that are given by

$$
Y_{l m}(\theta, \varphi)=\left[\frac{(2 l+1)(l-m) !}{4 \pi(l+m) !}\right]^{\frac{1}{2}} P_{l m}(\cos \theta) \exp (\operatorname{im} \varphi),
$$

where $P_{l m}(t)=\frac{(-1)^{m}}{2^{l} l !}\left(1-t^{2}\right)^{\frac{m}{2}} \frac{\mathrm{d}^{l+m}}{\mathrm{~d} t^{l+m}}\left(t^{2}-1\right)^{l}$ are associated Legendre polynomials. Note that in the case $m=0$, we simply write $P_{l}$ instead of $P_{l 0}$.

By $\widehat{f}_{n l m}^{\lambda}$ we denote the Fourier coefficients of the function $f \in L_{2}\left(\mathbb{R}^{3}, \omega_{\lambda}\right)$ with respect to the system $\mathcal{H}^{\lambda}$, i.e.,

$$
\widehat{f}_{n l m}^{\lambda}=\int_{0}^{\infty} \int_{0}^{\pi} \int_{0}^{2 \pi} f(r, \theta, \varphi) \overline{H_{n l m}^{\lambda}(r, \theta, \varphi)} \omega_{\lambda}(r) r^{2} \sin \theta \mathrm{d} \varphi \mathrm{d} \theta \mathrm{d} r .
$$

The following theorem holds.

THEOREM 2.1. [14] The system $\mathcal{H}^{\lambda}$ constitutes an orthonormal basis in the space $L_{2}\left(\mathbb{R}^{3}, \omega_{\lambda}\right)$, i.e., each function $f \in L_{2}\left(\mathbb{R}^{3}, \omega_{\lambda}\right)$ can be uniquely decomposed into the series

$$
f=\sum_{n=1}^{\infty}\left(\sum_{(l, m) \in \nabla n} \widehat{f}_{n l m}^{\lambda} H_{n l m}^{\lambda}\right)
$$

with the convergence in the sense of the space $L_{2}\left(\mathbb{R}^{3}, \omega_{\lambda}\right)$.

Although the authors in [14] consider only the case $\lambda=1$, the scale factor $\lambda$ does not influence on orthogonality and completeness of the system $\mathcal{H}^{\lambda}$.

As was mentioned in the introduction, the basis $\mathcal{H}^{\lambda}$ is called a spherical Gauss-Laguerre basis or shorter SGL basis and the coefficients $\widehat{f}_{n l m}^{\lambda}$ SGL Fourier coefficients of the function $f$. More detailed information about this basis can be found in [14] and [15].

3. Approximation of the function $\exp \left(-B|x|^{2}\right)$ in the space $L_{2}\left(\mathbb{R}^{3}, \omega_{\lambda}\right)$. In this section we investigate the behavior of the quantity $\mathcal{E}_{N}\left(g, \mathcal{H}^{\lambda}\right)$, where $g(\boldsymbol{x})=\exp \left(-B|\boldsymbol{x}|^{2}\right)$ with respect to the parameters $N \in \mathbb{N}$ and $\lambda>0$.

At first we formulate results regarding the SGL Fourier coefficients of the function $g$. 
THEOREM 3.1. The SGL Fourier coefficients of the function $g(\boldsymbol{x})=\exp \left(-B|\boldsymbol{x}|^{2}\right)$, $\boldsymbol{x} \in \mathbb{R}^{3}, B>0$, can be written as

$$
\widehat{g}_{n 00}^{\lambda}=\sqrt{\frac{2 \pi \Gamma\left(n+\frac{1}{2}\right)}{\Gamma(n)}} \frac{\lambda^{\frac{3}{4}}}{(1+B \lambda)^{\frac{3}{2}}}\left(\frac{B \lambda}{1+B \lambda}\right)^{n-1}, \quad \lambda>0,
$$

and $\widehat{g}_{n l m}^{\lambda}=0$ for $l \neq 0, m \neq 0$.

Proof. With $g(\boldsymbol{x})=g(r, \theta, \varphi)=\exp \left(-B r^{2}\right)$, we obtain

$$
\begin{aligned}
\widehat{g}_{n l m}^{\lambda} & =\int_{0}^{\infty} \int_{0}^{\pi} \int_{0}^{2 \pi} \exp \left(-B r^{2}\right) \overline{H_{n l m}^{\lambda}(r, \theta, \varphi)} \exp \left(-r^{2} / \lambda\right) r^{2} \sin \theta \mathrm{d} \varphi \mathrm{d} \theta \mathrm{d} r \\
& =\int_{0}^{\infty} \int_{0}^{\pi} \int_{0}^{2 \pi} \exp \left(-B r^{2}\right) R_{n l}^{\lambda}(r) \overline{Y_{l m}(\theta, \varphi)} \exp \left(-r^{2} / \lambda\right) r^{2} \sin \theta \mathrm{d} \varphi \mathrm{d} \theta \mathrm{d} r .
\end{aligned}
$$

Since

$$
\int_{0}^{\pi} \int_{0}^{2 \pi} \overline{Y_{l m}(\theta, \varphi)} \sin \theta \mathrm{d} \varphi \mathrm{d} \theta= \begin{cases}2 \sqrt{\pi}, & m=l=0 \\ 0, & \text { otherwise }\end{cases}
$$

we have that $\widehat{g}_{n l m}^{\lambda}=0$ if $l \neq 0, m \neq 0$.

Let now $n \in \mathbb{N}$ and $l=m=0$. After two changes of variables $\left(r / \sqrt{\lambda} \rightarrow t\right.$ and $\left.t^{2} \rightarrow y\right)$ we obtain

$$
\begin{aligned}
\left(\frac{8 \pi(n-1) !}{\lambda^{\frac{3}{2}} \Gamma\left(n+\frac{1}{2}\right)}\right)^{-\frac{1}{2}} \widehat{g}_{n 00}^{\lambda} & =\int_{0}^{\infty} \exp \left(-B r^{2}\right) L_{n-1}^{\frac{1}{2}}\left(r^{2} / \lambda\right) \exp \left(-r^{2} / \lambda\right) r^{2} \mathrm{~d} r \\
& =\lambda^{\frac{3}{2}} \int_{0}^{\infty} \exp \left(-t^{2}(1+B \lambda)\right) L_{n-1}^{\frac{1}{2}}\left(t^{2}\right) t^{2} \mathrm{~d} t \\
& =\frac{1}{2} \lambda^{\frac{3}{2}} \int_{0}^{\infty} \exp (-y(1+B \lambda)) L_{n-1}^{\frac{1}{2}}(y) y^{\frac{1}{2}} \mathrm{~d} y .
\end{aligned}
$$

Using formula [7, 7.414 (7)] for $\beta>-1$ and $s>0$

$$
\int_{0}^{\infty} \exp (-s t) t^{\beta} L_{n}^{(\alpha)}(t) \mathrm{d} t=\frac{\Gamma(\beta+1) \Gamma(\alpha+n+1)}{n ! \Gamma(\alpha+1)} s^{-\beta-1}{ }_{2} \mathrm{~F}_{1}(-n, \beta+1, \alpha+1,1 / s),
$$

where ${ }_{2} \mathrm{~F}_{1}$ is the hypergeometric function (see [1, Chap. 15]), we obtain

$$
\widehat{g}_{n 00}^{\lambda}=\sqrt{\frac{2 \pi \Gamma\left(n+\frac{1}{2}\right)}{(n-1) !}} \frac{\lambda^{\frac{3}{4}}}{(1+B \lambda)^{\frac{3}{2}}}{ }_{2} \mathrm{~F}_{1}\left(-n+1, \frac{3}{2}, \frac{3}{2}, \frac{1}{1+B \lambda}\right) .
$$

From the following property of the hypergeometric function ${ }_{2} \mathrm{~F}_{1}[1, \mathrm{p} .556]$

$$
{ }_{2} \mathrm{~F}_{1}(a, c, c, b)=(1-b)^{-a},
$$


we get that

$$
\widehat{g}_{n 00}^{\lambda}=\sqrt{\frac{2 \pi \Gamma\left(n+\frac{1}{2}\right)}{(n-1) !}} \frac{\lambda^{\frac{3}{4}}}{(1+B \lambda)^{\frac{3}{2}}}\left(1-\frac{1}{1+B \lambda}\right)^{n-1} .
$$

By using simple transformations and the definition of the Gamma function, we obtain the desired formula (3.1).

Note that we can express the asymptotic behavior of the coefficients $\widehat{g}_{n 00}^{\lambda}$ when $n \rightarrow \infty$ and $\lambda$ is fixed and vice versa when $\lambda \rightarrow \infty$ and $n$ is fixed.

Let at first $\lambda>0$ be fixed. According to the expansion (4.15) from [6] the quantity $\frac{\Gamma(n+1 / 2)}{\Gamma(n)}$ behaves at infinity (when $\left.n \rightarrow \infty\right)$ as

$$
\frac{\Gamma\left(n+\frac{1}{2}\right)}{\Gamma(n)} \sim n^{\frac{1}{2}} .
$$

From this we have that

$$
\widehat{g}_{n 00}^{\lambda} \sim C_{1}(\lambda, B) n^{\frac{1}{4}}\left(\frac{B \lambda}{1+B \lambda}\right)^{n-1}, n \rightarrow \infty,
$$

where $C_{1}(\lambda, B)=\frac{\sqrt{2 \pi} \lambda^{\frac{3}{4}}}{(1+B \lambda)^{\frac{3}{2}}}$.

Let now $n \in \mathbb{N}$ be fixed and $\lambda \rightarrow \infty$. From (3.1) it is easy to see that in this case

$$
\widehat{g}_{n 00}^{\lambda} \sim C_{2}(n, B) \lambda^{-\frac{3}{4}},
$$

where $C_{2}(n, B)=B^{-\frac{3}{4}} \sqrt{\frac{2 \pi \Gamma(n+1 / 2)}{\Gamma(n)}}$.

Now we are ready to prove the following results on the error of approximation $\mathcal{E}_{N}\left(g, \mathcal{H}^{\lambda}\right)$.

THEOREM 3.2. For the function $g(\boldsymbol{x})=\mathrm{e}^{-B|\boldsymbol{x}|^{2}}, \boldsymbol{x} \in \mathbb{R}^{3}, B>0$, and $\lambda>0, N \in \mathbb{N}$, we have

$$
\begin{aligned}
\mathcal{E}_{N}\left(g, \mathcal{H}^{\lambda}\right)= & \sqrt{\frac{2 \pi \Gamma\left(N+\frac{3}{2}\right)}{\Gamma(N+1)} \frac{\lambda^{\frac{3}{4}}}{(1+B \lambda)^{\frac{3}{2}}}\left(\frac{B \lambda}{1+B \lambda}\right)^{N}} \\
& \times \sqrt{{ }_{2} F_{1}\left(1, N+\frac{3}{2}, N+1 ;\left(\frac{B \lambda}{1+B \lambda}\right)^{2}\right)} .
\end{aligned}
$$

Proof. Applying Parseval's equality, we obtain

$$
\begin{aligned}
\mathcal{E}_{N}^{2}\left(g, \mathcal{H}^{\lambda}\right) & =\left\|g-\sum_{n=1}^{N}\left(\sum_{(l, m) \in \nabla n} \widehat{g}_{n l m}^{\lambda} H_{n l m}^{\lambda}\right)\right\|_{L_{2}\left(\mathbb{R}^{3}, \omega_{\lambda}\right)}^{2} \\
& =\sum_{n=N+1}^{\infty}\left(\sum_{(l, m) \in \nabla n}\left|\widehat{g}_{n l m}^{\lambda}\right|^{2}\right) .
\end{aligned}
$$

Substituting the SGL Fourier coefficients (3.1) in (3.3) we get

$$
\begin{aligned}
\mathcal{E}_{N}^{2}\left(g, \mathcal{H}^{\lambda}\right) & =\sum_{n=N+1}^{\infty}\left|\widehat{g}_{n 00}^{\lambda}\right|^{2}=2 \pi\left(\frac{\lambda^{\frac{3}{4}}}{(1+B \lambda)^{\frac{3}{2}}}\right)^{2} \sum_{n=N+1}^{\infty} \frac{\Gamma\left(n+\frac{1}{2}\right)}{\Gamma(n)}\left(\frac{B \lambda}{1+B \lambda}\right)^{2(n-1)} \\
& =2 \pi\left(\frac{\lambda^{\frac{3}{4}}}{(1+B \lambda)^{\frac{3}{2}}}\right)^{2}\left(\frac{B \lambda}{1+B \lambda}\right)^{2 N} \sum_{k=0}^{\infty} \frac{\Gamma\left(k+N+\frac{3}{2}\right)}{\Gamma(k+N+1)}\left(\frac{B \lambda}{1+B \lambda}\right)^{2 k} .
\end{aligned}
$$


Using the expansion of the hypergeometric function ${ }_{2} F_{1}(1.4)$

$$
\sum_{k=0}^{\infty} \frac{\Gamma(k+a)}{\Gamma(k+b)} x^{k}=\frac{\Gamma(a)}{\Gamma(b)}{ }_{2} F_{1}(1, a, b ; x),|x|<1,
$$

we obtain the necessary equality.

Before formulation of the next theorem we give a proof of one property of the hypergeometric function that we will use few times.

LEMMA 3.3. For the hypergeometric function ${ }_{2} F_{1}(1, b, c ; t)$, the following representation

$$
\begin{aligned}
{ }_{2} F_{1}(1, b, c ; t)= & \frac{\Gamma(c) \Gamma(b-c+1)}{\Gamma(b)} t^{1-c}(1-t)^{c-b-1} \\
& -t^{1-c} \sum_{k=0}^{c-2} \frac{(-1)^{k}(c-1) !}{k !(c-2-k) !} \frac{(1-t)^{k}}{b-c+1+k},
\end{aligned}
$$

holds for $c \in \mathbb{N}$ and $0<t<1$.

Proof. Let

$$
\mathbf{B}_{z}(a, b)=\int_{0}^{z} t^{a-1}(1-t)^{b-1} \mathrm{~d} t, a, b>0,|z|<1,
$$

be the incomplete beta function $[7,8.39]$. By using transformation formulas for the hypergeometric function ${ }_{2} F_{1}[7,9.131]$

$$
{ }_{2} F_{1}(a, b, c ; t)=(1-t)^{c-b-a}{ }_{2} F_{1}(c-a, c-b, c ; t),
$$

we obtain

$$
{ }_{2} F_{1}(1, b, c ; t)=(1-t)^{c-b-1}{ }_{2} F_{1}(c-1, c-b, c ; t) .
$$

Applying the following formula [7, 8.391]

$$
{ }_{2} F_{1}(p, 1-q, p+1 ; t)=p t^{-p} \mathbf{B}_{t}(p, q)
$$

with $p=c-1$ and $q=b-c+1$, we get

$$
{ }_{2} F_{1}(1, b, c ; t)=(c-1) t^{1-c}(1-t)^{c-b-1} \mathbf{B}_{t}(c-1, b-c+1) .
$$

Finally, from $[1,6.6 .2]$ and $[1,26.5 .6]$ we have

$$
\mathbf{B}_{t}(n, \beta)=\mathbf{B}(n, \beta)-(1-t)^{\beta} \sum_{k=0}^{n-1} \frac{(-1)^{k}(n-1) !}{k !(n-1-k) !} \frac{(1-t)^{k}}{\beta+k}, n \in \mathbb{N},
$$

where $\mathbf{B}(\alpha, \beta)=\frac{\Gamma(\alpha) \Gamma(\beta)}{\Gamma(\alpha+\beta)}$ is the beta function [7, 8.384 (1)], which implies (3.5).

The next theorem describes the asymptotic behavior of the quantity $\mathcal{E}_{N}\left(g, \mathcal{H}^{\lambda}\right)$ with respect to the parameter $N \in \mathbb{N}$ for fixed $\lambda, B>0$; see Figure 3.1, left.

THEOREM 3.4. The quantity $\mathcal{E}_{N}\left(g, \mathcal{H}^{\lambda}\right)$ for $g(\boldsymbol{x})=\exp \left(-B|\boldsymbol{x}|^{2}\right), B, \lambda>0$, behaves asymptotically like

$$
\mathcal{E}_{N}\left(g, \mathcal{H}^{\lambda}\right) \sim C_{3}(\lambda, B)(N+1)^{\frac{1}{4}}\left(\frac{B \lambda}{1+B \lambda}\right)^{N}, N \rightarrow \infty,
$$


where $C_{3}(\lambda, B)=\frac{\sqrt{2 \pi} \lambda^{3 / 4}}{(1+B \lambda)^{3 / 2}} C_{4}(\lambda, B)$ and

$$
\frac{1+B \lambda}{\sqrt{1+2 B \lambda}}<C_{4}(\lambda, B) \leq \sqrt{\frac{2}{3} \frac{(1+B \lambda)^{3}-(1+2 B \lambda)^{\frac{3}{2}}}{(B \lambda)^{2} \sqrt{1+2 B \lambda}}} \frac{1+B \lambda}{\sqrt{1+2 B \lambda}} .
$$

Proof. To prove (3.7) we use Theorem 3.2 together with (3.2) and the boundedness of the function ${ }_{2} F_{1}\left(1, N+\frac{3}{2}, N+1 ;\left(\frac{B \lambda}{1+B \lambda}\right)^{2}\right)$ with respect to the parameter $N$ that we show below.

From (3.2) we have

$$
\sqrt{\frac{\Gamma\left(N+\frac{3}{2}\right)}{\Gamma(N+1)}} \sim(N+1)^{\frac{1}{4}}, N \rightarrow \infty .
$$

Let us further prove that ${ }_{2} F_{1}\left(1, N+\frac{3}{2}, N+1 ;\left(\frac{B \lambda}{1+B \lambda}\right)^{2}\right)$ is bounded with respect to $N \in \mathbb{N}$ for all fixed positive values $B$ and $\lambda$. For an arbitrary $N \in \mathbb{N}$, we consider a sequence $a_{N}:={ }_{2} F_{1}\left(1, N+\frac{3}{2}, N+1 ; t\right), 0<t<1$ and prove that $a_{N}>a_{N+1}$ for all $N \in \mathbb{N}$.

According to (3.4) we obtain

$$
\begin{aligned}
a_{N}-a_{N+1} & ={ }_{2} F_{1}\left(1, N+\frac{3}{2}, N+1 ; t\right)-{ }_{2} F_{1}\left(1, N+\frac{5}{2}, N+2 ; t\right) \\
& =\frac{\Gamma(N+1)}{\Gamma\left(N+\frac{3}{2}\right)} \sum_{k=0}^{\infty} \frac{\Gamma\left(k+N+\frac{3}{2}\right)}{\Gamma(k+N+1)} t^{k}-\frac{\Gamma(N+2)}{\Gamma\left(N+\frac{5}{2}\right)} \sum_{k=0}^{\infty} \frac{\Gamma\left(k+N+\frac{5}{2}\right)}{\Gamma(k+N+2)} t^{k} .
\end{aligned}
$$

Let us denote $f(N)=\frac{\Gamma\left(N+\frac{5}{2}\right)}{\Gamma(N+1)}\left(a_{N}-a_{N+1}\right)$. Then

$$
f(N)=\frac{\Gamma\left(N+\frac{5}{2}\right)}{\Gamma\left(N+\frac{3}{2}\right)} \sum_{k=0}^{\infty} \frac{\Gamma\left(k+N+\frac{3}{2}\right)}{\Gamma(k+N+1)} t^{k}-\frac{\Gamma(N+2)}{\Gamma(N+1)} \sum_{k=0}^{\infty} \frac{\Gamma\left(k+N+\frac{5}{2}\right)}{\Gamma(k+N+2)} t^{k} .
$$

From the following property of the Gamma function

$$
\Gamma\left(n+\frac{1}{2}\right)=\frac{(2 n) !}{4^{n} n !} \sqrt{\pi}, n \in \mathbb{N},
$$

we have that

$$
\frac{\Gamma\left(N+\frac{3}{2}\right)}{\Gamma\left(N+\frac{5}{2}\right)}=\frac{2}{2 N+3}
$$

By using this equality, we get

$$
\begin{aligned}
f(N) & =\frac{2 N+3}{2} \sum_{k=0}^{\infty} \frac{\Gamma\left(k+N+\frac{3}{2}\right)}{\Gamma(k+N+1)} t^{k}-(N+1) \sum_{k=0}^{\infty} \frac{\Gamma\left(k+N+\frac{5}{2}\right)}{\Gamma(k+N+2)} t^{k} \\
& =\sum_{k=0}^{\infty} \frac{\Gamma\left(k+N+\frac{5}{2}\right)}{\Gamma(k+N+1)}\left(\left(N+\frac{3}{2}\right) \frac{\Gamma\left(k+N+\frac{3}{2}\right)}{\Gamma\left(k+N+\frac{5}{2}\right)}-(N+1) \frac{\Gamma(k+N+1)}{\Gamma(k+N+2)}\right) t^{k} \\
& =\sum_{k=0}^{\infty} \frac{\Gamma\left(k+N+\frac{5}{2}\right)}{\Gamma(k+N+1)} \frac{k t^{k}}{(2 N+3+2 k)(N+1+k)}
\end{aligned}
$$


which implies that $f(N)>0$ and consequently $a_{N}>a_{N+1}, N \in \mathbb{N}$. From this, for all $N \in \mathbb{N}$, we have

$$
\begin{aligned}
{ }_{2} F_{1}\left(1, N+\frac{3}{2}, N+1 ;\left(\frac{B \lambda}{1+B \lambda}\right)^{2}\right) & \leq{ }_{2} F_{1}\left(1, \frac{5}{2}, 2,\left(\frac{B \lambda}{1+B \lambda}\right)^{2}\right) \\
& =\frac{2}{3} \frac{(1+B \lambda)^{3}-(1+2 B \lambda)^{\frac{3}{2}}}{(B \lambda)^{2} \sqrt{1+2 B \lambda}} \frac{(1+B \lambda)^{2}}{1+2 B \lambda} .
\end{aligned}
$$

The last equality is due to (3.5). On the other hand, by using (3.9) we get

$$
\begin{aligned}
{ }_{2} F_{1}\left(1, N+\frac{3}{2}, N+1 ;\left(\frac{B \lambda}{1+B \lambda}\right)^{2}\right) & =\frac{\Gamma(N+1)}{\Gamma\left(N+\frac{3}{2}\right)} \sum_{k=0}^{\infty} \frac{\Gamma\left(k+N+\frac{3}{2}\right)}{\Gamma(k+N+1)}\left(\frac{B \lambda}{1+B \lambda}\right)^{2 k} \\
& =1+\sum_{k=1}^{\infty} \frac{(2 N+2 k+1) ! !}{(2 N+2 k) ! !}\left(\frac{B \lambda}{1+B \lambda}\right)^{2 k} \\
& >\sum_{k=0}^{\infty}\left(\frac{B \lambda}{1+B \lambda}\right)^{2 k}=\frac{(1+B \lambda)^{2}}{1+2 B \lambda} .
\end{aligned}
$$

From (3.11) and (3.12) we have that for all $N \in \mathbb{N}$

$$
\frac{(1+B \lambda)^{2}}{1+2 B \lambda}<C_{4}^{2}(\lambda, B) \leq \frac{2}{3} \frac{(1+B \lambda)^{3}-(1+2 B \lambda)^{\frac{3}{2}}}{(B \lambda)^{2} \sqrt{1+2 B \lambda}} \frac{(1+B \lambda)^{2}}{1+2 B \lambda} .
$$

From relations (3.8), (3.13) and Theorem 3.2 we get the asymptotic (3.7).
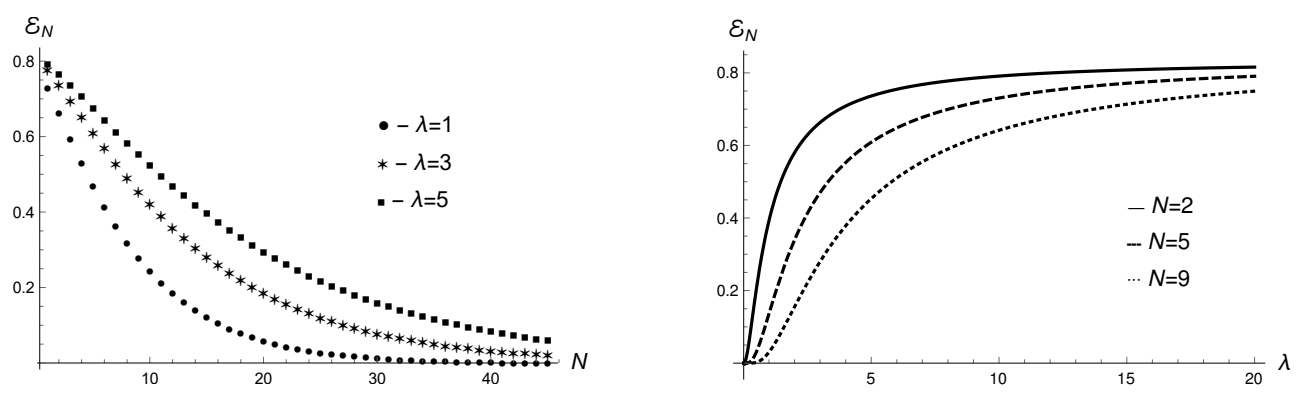

FIG. 3.1. Graphics of the behavior of $\mathcal{E}_{N}\left(g, \mathcal{H}^{\lambda}\right)(B=2)$ with respect to $N$ (left) and with respect to $\lambda$ (right).

The next theorem describes the behavior of the quantity $\mathcal{E}_{N}\left(g, \mathcal{H}^{\lambda}\right)$ with respect to the parameter $\lambda>0$ for fixed $N \in \mathbb{N}$ and $B>0$; see Figure 3.1, right.

THEOREM 3.5. The quantity $\mathcal{E}_{N}\left(g, \mathcal{H}^{\lambda}\right)$ for $g(\boldsymbol{x})=\exp \left(-B|\boldsymbol{x}|^{2}\right)$, as a function of $\lambda$, $\lambda>0$, is increasing on the segment $(0,+\infty)$ for all $N \in \mathbb{N}$. Moreover,

$$
\lim _{\lambda \rightarrow \infty} \mathcal{E}_{N}\left(g, \mathcal{H}^{\lambda}\right)=\left(\frac{\pi}{2 B}\right)^{\frac{3}{4}}, N \in \mathbb{N} .
$$

Proof. At first we prove (3.14). Let us find

$$
J:=\lim _{\lambda \rightarrow \infty} \frac{\lambda^{\frac{3}{2}}}{(1+B \lambda)^{3}}{ }_{2} F_{1}\left(1, N+\frac{3}{2}, N+1 ;\left(\frac{B \lambda}{1+B \lambda}\right)^{2}\right) .
$$


To this end we use the following limit (see Theorem 2.1.3 [2, p. 63])

$$
\lim _{z \rightarrow 1}(1-z)_{2}^{a+b-c} F_{1}(a, b, c ; z)=\frac{\Gamma(c) \Gamma(a+b-c)}{\Gamma(a) \Gamma(b)}, c-b-a<0 .
$$

From (3.16) taking into account that $\left(\frac{B \lambda}{1+B \lambda}\right)^{2} \rightarrow 1$ as $\lambda \rightarrow \infty$, we have

$$
\lim _{\lambda \rightarrow \infty}\left(1-\left(\frac{B \lambda}{1+B \lambda}\right)^{2}\right)^{\frac{3}{2}}{ }_{2} F_{1}\left(1, N+\frac{3}{2}, N+1 ;\left(\frac{B \lambda}{1+B \lambda}\right)^{2}\right)=\frac{\Gamma(N+1) \Gamma\left(\frac{3}{2}\right)}{\Gamma\left(N+\frac{3}{2}\right)} .
$$

By using (3.17) we get for (3.15)

$$
\begin{aligned}
J & =\lim _{\lambda \rightarrow \infty} \frac{\lambda^{\frac{3}{2}}}{(1+B \lambda)^{3}}\left(1-\left(\frac{B \lambda}{1+B \lambda}\right)^{2}\right)^{-\frac{3}{2}} \\
& \times\left(1-\left(\frac{B \lambda}{1+B \lambda}\right)^{2}\right)^{\frac{3}{2}}{ }_{2} F_{1}\left(1, N+\frac{3}{2}, N+1 ;\left(\frac{B \lambda}{1+B \lambda}\right)^{2}\right) \\
& =\frac{\Gamma(N+1) \Gamma\left(\frac{3}{2}\right)}{\Gamma\left(N+\frac{3}{2}\right)} \lim _{\lambda \rightarrow \infty} \frac{\lambda^{\frac{3}{2}}}{(1+2 B \lambda)^{\frac{3}{2}}}=\frac{\Gamma(N+1) \Gamma\left(\frac{3}{2}\right)}{\Gamma\left(N+\frac{3}{2}\right)}\left(\frac{1}{2 B}\right)^{\frac{3}{2}} .
\end{aligned}
$$

From (3.15), (3.18) and Theorem 3.2, using that $\Gamma\left(\frac{3}{2}\right)=\frac{1}{2} \sqrt{\pi}$, we obtain

$$
\lim _{\lambda \rightarrow \infty} \mathcal{E}_{N}\left(g, \mathcal{H}^{\lambda}\right)=\sqrt{\frac{2 \pi \Gamma\left(N+\frac{3}{2}\right)}{\Gamma(N+1)}} \sqrt{\frac{\Gamma(N+1) \Gamma\left(\frac{3}{2}\right)}{\Gamma\left(N+\frac{3}{2}\right)}}\left(\frac{1}{2 B}\right)^{\frac{3}{4}}=\left(\frac{\pi}{2 B}\right)^{\frac{3}{4}},
$$

for all $N \in \mathbb{N}$.

Our further goal is to prove that $\mathcal{E}_{N}\left(g, \mathcal{H}^{\lambda}\right)$ is increasing for $\lambda \in(0 ;+\infty)$. Let us first find $\frac{\partial\left(\mathcal{E}_{N}\left(g, \mathcal{H}^{\lambda}\right)\right)}{\partial \lambda}$. Note that a derivative of the hypergeometric function ${ }_{2} F_{1}$ can be found by the formula $[1,15.2 .1]$ :

$$
\frac{\partial\left({ }_{2} F_{1}(a, b, c ; x)\right)}{\partial x}=\frac{a b}{c}{ }_{2} F_{1}(a+1, b+1, c+1 ; x) .
$$

Taking this into account, we get

$$
\begin{aligned}
\frac{\partial\left(\mathcal{E}_{N}\left(g, \mathcal{H}^{\lambda}\right)\right)}{\partial \lambda}= & \sqrt{\frac{2 \pi \Gamma\left(N+\frac{3}{2}\right)}{\Gamma(N+1)}\left(\frac{B \lambda}{1+B \lambda}\right)^{N-1}} \\
& \times \frac{B \lambda^{\frac{3}{4}} \times R(N, \lambda)}{4(1+B \lambda)^{\frac{7}{2}} \sqrt{{ }_{2} F_{1}\left(1, N+\frac{3}{2}, N+1 ;\left(\frac{B \lambda}{1+B \lambda}\right)^{2}\right)}}
\end{aligned}
$$

where

$$
\begin{aligned}
R(N, \lambda):= & (3+4 N-3 B \lambda){ }_{2} F_{1}\left(1, N+\frac{3}{2}, N+1 ;\left(\frac{B \lambda}{1+B \lambda}\right)^{2}\right) \\
& +4\left(\frac{B \lambda}{1+B \lambda}\right)^{2} \frac{N+\frac{3}{2}}{N+1}{ }_{2} F_{1}\left(2, N+\frac{5}{2}, N+2 ;\left(\frac{B \lambda}{1+B \lambda}\right)^{2}\right)
\end{aligned}
$$


and we prove that $R(N, \lambda)>0$ for $\lambda>0$ and all choices of parameters $N$ and $B$.

For simplicity we let $\frac{B \lambda}{1+B \lambda}=t$. Then we get a new function $\mathrm{R}$ for $0<t<1$

$$
\begin{aligned}
\mathrm{R}(N, t)= & \left(3+4 N-\frac{3 t}{1-t}\right){ }_{2} F_{1}\left(1, N+\frac{3}{2}, N+1 ; t^{2}\right) \\
& +4 t^{2} \frac{N+\frac{3}{2}}{N+1}{ }_{2} F_{1}\left(2, N+\frac{5}{2}, N+2 ; t^{2}\right) .
\end{aligned}
$$

By using the following expansions of the hypergeometric functions

$$
\begin{aligned}
{ }_{2} F_{1}\left(2, N+\frac{5}{2}, N+2 ; t^{2}\right) & =\sum_{k=0}^{\infty} \frac{(2)_{k}\left(N+\frac{5}{2}\right)_{k}}{(N+2)_{k} k !} t^{2 k} \\
& =\frac{\Gamma(N+2)}{\Gamma\left(N+\frac{5}{2}\right)} \sum_{k=0}^{\infty}(k+1) \frac{\Gamma\left(k+N+\frac{5}{2}\right)}{\Gamma(k+N+2)} t^{2 k} \\
& =\frac{\Gamma(N+2)}{\Gamma\left(N+\frac{5}{2}\right)} t^{-2} \sum_{k=0}^{\infty} k \frac{\Gamma\left(k+N+\frac{3}{2}\right)}{\Gamma(k+N+1)} t^{2 k} \\
{ }_{2} F_{1}\left(1, N+\frac{3}{2}, N+1 ; t^{2}\right) & =\frac{\Gamma(N+1)}{\Gamma\left(N+\frac{3}{2}\right)} \sum_{k=0}^{\infty} \frac{\Gamma\left(k+N+\frac{3}{2}\right)}{\Gamma(k+N+1)} t^{2 k}
\end{aligned}
$$

and (3.10), we obtain that

$$
\begin{aligned}
\mathrm{R}(N, t)= & \left(3+4 N-\frac{3 t}{1-t}\right) \frac{\Gamma(N+1)}{\Gamma\left(N+\frac{3}{2}\right)} \sum_{k=0}^{\infty} \frac{\Gamma\left(k+N+\frac{3}{2}\right)}{\Gamma(k+N+1)} t^{2 k} \\
& +4 \frac{N+\frac{3}{2}}{N+1} \frac{\Gamma(N+2)}{\Gamma\left(N+\frac{5}{2}\right)} \sum_{k=0}^{\infty} k \frac{\Gamma\left(k+N+\frac{3}{2}\right)}{\Gamma(k+N+1)} t^{2 k} \\
= & \frac{\Gamma(N+1)}{\Gamma\left(N+\frac{3}{2}\right)} \sum_{k=0}^{\infty} \frac{\Gamma\left(k+N+\frac{3}{2}\right)}{\Gamma(k+N+1)}\left(3+4 N-\frac{3 t}{1-t}+4 k\right) t^{2 k}
\end{aligned}
$$

Let us consider the series

$$
\sum_{k=0}^{\infty} \frac{\Gamma\left(k+N+\frac{3}{2}\right)}{\Gamma(k+N+1)}\left(3+4 N-\frac{3 t}{1-t}+4 k\right) t^{2 k} .
$$

By $S(N, t)$ we denote the sum of the series (3.21) and we prove by induction with respect to $N$ that $S(N, t)>0$ for all $0<t<1$.

Let $N=1$. Then from (3.19) and (3.20) by using (3.6), we get

$$
\begin{aligned}
S(1, t) & =\frac{\Gamma\left(\frac{5}{2}\right)}{\Gamma(2)} \mathrm{R}(1, t)=\frac{3 \sqrt{\pi}}{4}\left(\frac{7-10 t}{1-t}{ }_{2} F_{1}\left(1, \frac{5}{2}, 2 ; t^{2}\right)+5 t^{2}{ }_{2} F_{1}\left(2, \frac{7}{2}, 3 ; t^{2}\right)\right) \\
& =\frac{3 \sqrt{\pi}}{4}\left(\frac{7-10 t}{1-t}{ }_{2} F_{1}\left(1, \frac{5}{2}, 2 ; t^{2}\right)+\frac{5 t^{2}}{\left(1-t^{2}\right)^{\frac{5}{2}}}{ }_{2} F_{1}\left(1,-\frac{1}{2}, 3 ; t^{2}\right)\right) .
\end{aligned}
$$

By using (3.5) we continue

$$
\begin{aligned}
S(1, t) & =\frac{3 \sqrt{\pi}}{4}\left(\frac{7-10 t}{1-t} \frac{2\left(1-\left(1-t^{2}\right)^{\frac{3}{2}}\right)}{3 t^{2}\left(1-t^{2}\right)^{\frac{3}{2}}}+5 t^{2} \frac{4\left(-2+5 t^{2}+2\left(1-t^{2}\right)^{\frac{5}{2}}\right)}{15 t^{4}\left(1-t^{2}\right)^{\frac{5}{2}}}\right) \\
& =\frac{3 \sqrt{\pi}\left((2 t+3) t^{2} \sqrt{1-t^{2}}+1-\sqrt{1-t^{2}}\right)}{2(1-t) t^{2}(1+t)^{2} \sqrt{1-t^{2}}} .
\end{aligned}
$$


Since $(2 t+3) t^{2} \sqrt{1-t^{2}}+1>1$ and $\sqrt{1-t^{2}}<1$ for $0<t<1$, we have that $(2 t+3) t^{2} \sqrt{1-t^{2}}+1-\sqrt{1-t^{2}}>0$ and consequently $S(1, t)>0,0<t<1$.

Further we assume that for $N \in \mathbb{N}$ the sum $S(N, t)$ is positive and prove that $S(N+1, t)$ is also positive. From (3.10) and the definition of the Gamma function we have

$$
\begin{aligned}
S(N+1, t) & =\sum_{k=0}^{\infty} \frac{\Gamma\left(k+N+\frac{5}{2}\right)}{\Gamma(k+N+2)}\left(3+4(N+1)-\frac{3 t}{1-t}+4 k\right) t^{2 k} \\
& =\sum_{k=0}^{\infty} \frac{\Gamma\left(k+N+\frac{3}{2}\right)\left(k+N+\frac{3}{2}\right)}{\Gamma(k+N+1)(k+N+1)}\left(3+4(N+1)-\frac{3 t}{1-t}+4 k\right) t^{2 k}
\end{aligned}
$$

By using simple transformations, we proceed as follows

$$
\begin{aligned}
S(N+1, t)= & \sum_{k=0}^{\infty} \frac{\Gamma\left(k+N+\frac{3}{2}\right)}{\Gamma(k+N+1)}\left(1+\frac{1}{2 k+2 N+2}\right)\left(7+4 N-\frac{3 t}{1-t}+4 k\right) t^{2 k} \\
= & \sum_{k=0}^{\infty} \frac{\Gamma\left(k+N+\frac{3}{2}\right)}{\Gamma(k+N+1)}\left(3+4 N-\frac{3 t}{1-t}+4 k\right) t^{2 k} \\
& +\sum_{k=0}^{\infty} \frac{\Gamma\left(k+N+\frac{3}{2}\right)}{\Gamma(k+N+1)}\left(6+\frac{3}{2} \frac{\frac{1-2 t}{1-t}}{k+N+1}\right) t^{2 k} \\
= & S(N, t)+\sigma(N, t),
\end{aligned}
$$

where

$$
\sigma(N, t):=\sum_{k=0}^{\infty} \frac{\Gamma\left(k+N+\frac{3}{2}\right)}{\Gamma(k+N+1)}\left(6+\frac{3}{2} \frac{\frac{1-2 t}{1-t}}{k+N+1}\right) t^{2 k} .
$$

If $0<t \leq 1 / 2$, then it is obvious that $\sigma(N, t)>0$ for all $N \in \mathbb{N}$. Let now $1 / 2<t<1$. The sum $\sigma(N, t)$ can be rewritten in the following way

$$
\sigma(N, t)=6 \sum_{k=0}^{\infty} \frac{\Gamma\left(k+N+\frac{3}{2}\right)}{\Gamma(k+N+1)} t^{2 k}-\frac{3(2 t-1)}{2(1-t)} \sum_{k=0}^{\infty} \frac{\Gamma\left(k+N+\frac{3}{2}\right)}{\Gamma(k+N+2)} t^{2 k} .
$$

Let us further prove that $\sigma(N+1, t)>\sigma(N, t), N \in \mathbb{N}$. By $h(N)$ we denote $h(N)=\sigma(N+1, t)-\sigma(N, t), \frac{1}{2}<t<1$, and prove that $h(N)>0$ for all $N \in \mathbb{N}$. We have

$$
\begin{aligned}
h(N)= & \sigma(N+1, t)-\sigma(N, t) \\
= & 6 \sum_{k=0}^{\infty} \frac{\Gamma\left(k+N+\frac{3}{2}\right)}{\Gamma(k+N+1)}\left(\frac{k+N+\frac{3}{2}}{k+N+1}-1\right) t^{2 k} \\
& +\frac{3(2 t-1)}{2(1-t)} \sum_{k=0}^{\infty} \frac{\Gamma\left(k+N+\frac{3}{2}\right)}{\Gamma(k+N+2)}\left(1-\frac{k+N+\frac{3}{2}}{k+N+2}\right) t^{2 k} \\
= & 6 \sum_{k=0}^{\infty} \frac{\Gamma\left(k+N+\frac{3}{2}\right)}{\Gamma(k+N+1)} \frac{\frac{1}{2}}{k+N+1} t^{2 k} \\
& +\frac{3(2 t-1)}{2(1-t)} \sum_{k=0}^{\infty} \frac{\Gamma\left(k+N+\frac{3}{2}\right)}{\Gamma(k+N+2)} \frac{\frac{1}{2}}{k+N+2} t^{2 k} .
\end{aligned}
$$


From (3.24) we see that $h(N)>0$ for all $N \in \mathbb{N}$. Therefore, $\sigma(N, t) \geq \sigma(1, t)$ for all $N \in \mathbb{N}$. By using (3.4) and (3.23), we obtain

$$
\sigma(1, t)=6 \frac{\Gamma\left(\frac{5}{2}\right)}{\Gamma(2)}{ }_{2} F_{1}\left(1, \frac{5}{2}, 2 ; t^{2}\right)-\frac{3(2 t-1)}{2(1-t)} \frac{\Gamma\left(\frac{5}{2}\right)}{\Gamma(3)}{ }_{2} F_{1}\left(1, \frac{5}{2}, 3 ; t^{2}\right)
$$

By using (3.5) and simple transformations, we have

$$
\begin{aligned}
\sigma(1, t) & =\frac{3 \sqrt{\pi}\left(1-\left(1-t^{2}\right) \sqrt{1-t^{2}}\right)}{t^{2}\left(1-t^{2}\right) \sqrt{1-t^{2}}}-\frac{3 \sqrt{\pi}(2 t-1)\left(2-\left(2+t^{2}\right) \sqrt{1-t^{2}}\right)}{4(1-t) t^{4} \sqrt{1-t^{2}}} \\
& =\frac{3 \sqrt{\pi}\left(2+\left(2 t+t^{3}+6 t^{4}\right) \sqrt{1-t^{2}}-\left(2 t+\left(2+t^{2}\right) \sqrt{1-t^{2}}\right)\right)}{4 t^{4}\left(1-t^{2}\right)^{\frac{3}{2}}}
\end{aligned}
$$

Our further goal is to prove that for $\frac{1}{2}<t<1$

$$
2+\left(2 t+t^{3}+6 t^{4}\right) \sqrt{1-t^{2}}>2 t+\left(2+t^{2}\right) \sqrt{1-t^{2}},
$$

which follows from

$$
2-2 t>\left(2-2 t+t^{2}-t^{3}\right) \sqrt{1-t^{2}}=(1-t)\left(2+t^{2}\right) \sqrt{1-t^{2}},
$$

which for $0<t<1$ is equivalent to $4>4-3 t^{4}-t^{6}$.

From this and (3.25) we have that $\sigma(1, t)>0$ for $\frac{1}{2}<t<1$. Since $\sigma(N, t)>\sigma(1, t)>0$ that together with the assumption $S(N, t)>0$ and (3.22) implies $S(N+1, t)>0$.

4. Approximation of the function $\exp \left(-B\left|x-x_{0}\right|^{2}\right)$ in the space $L_{2}\left(\mathbb{R}^{3}, \omega_{\lambda}\right)$. In this section we prove formulas that show the behavior of SGL Fourier coefficients of Gaussian functions $g\left(\boldsymbol{x}-\boldsymbol{x}_{0}\right)=\exp \left(-B\left|\boldsymbol{x}-\boldsymbol{x}_{0}\right|^{2}\right)$ in the space $L_{2}\left(\mathbb{R}^{3}, \omega_{\lambda}\right)$ and present numerical results with respect to the behavior of the quantity $\mathcal{E}_{N}\left(g, \mathcal{H}^{\lambda}\right)$ for these functions. We consider two cases in the spherical coordinates: I. $\boldsymbol{x}_{0}=\left(r_{0}, 0,0\right)$; II. $\boldsymbol{x}_{0}=\left(r_{0}, \theta_{0}, \varphi_{0}\right)$.

Further we use the following notations: Let

$$
p_{\lambda, r_{0}}:=\frac{2 B \sqrt{\lambda} r_{0}}{\sqrt{2 B \lambda+2}}, \quad C_{n l}^{\lambda}:=\left[\frac{2 \pi^{2} \lambda^{\frac{3}{2}}(2 l+1)(n-l-1) !}{\Gamma\left(n+\frac{1}{2}\right)}\right]^{\frac{1}{2}},
$$

and by $\Phi_{l, j, i}(t), t \geq 0$, we denote

$$
\begin{aligned}
\Phi_{l, j, i}(t):= & \frac{1}{(l-2 i+1) \Gamma\left(\frac{l+2 j+4}{2}\right)}{ }_{2} F_{2}\left(\frac{l-2 i+1}{2}, \frac{l+2 j+3}{2} ; \frac{1}{2}, \frac{l-2 i+3}{2} ; \frac{t^{2}}{2}\right) \\
& -\frac{\sqrt{2} t}{(l-2 i+2) \Gamma\left(\frac{l+2 j+3}{2}\right)}{ }_{2} F_{2}\left(\frac{l-2 i+2}{2}, \frac{l+2 j+4}{2} ; \frac{3}{2}, \frac{l-2 i+4}{2} ; \frac{t^{2}}{2}\right),
\end{aligned}
$$

where ${ }_{2} F_{2}$ is the generalized hypergeometric function [7, 9.14] defined by

$$
{ }_{2} F_{2}\left(a_{1}, a_{2} ; b_{1}, b_{2} ; t\right)=\sum_{k=0}^{\infty} \frac{\left(a_{1}\right)_{k}\left(a_{2}\right)_{k} z^{k}}{\left(b_{1}\right)_{k}\left(b_{2}\right)_{k} k !} .
$$


I. Let first $\boldsymbol{x}_{0}=\left(r_{0}, 0,0\right)$.

THEOREM 4.1. For the function $g\left(\boldsymbol{x}-\boldsymbol{x}_{0}\right)=\exp \left(-B\left|\boldsymbol{x}-\boldsymbol{x}_{0}\right|^{2}\right), \boldsymbol{x}, \boldsymbol{x}_{0} \in \mathbb{R}^{3}, \boldsymbol{x}_{0}=$ $\left(r_{0}, 0,0\right), B>0$, and $\lambda>0$, we have

$$
\begin{aligned}
\hat{g}_{n l 0}^{\lambda}= & \exp \left(-B r_{0}^{2}\right) C_{n l}^{\lambda} \sum_{j=0}^{n-l-1} \frac{(-1)^{j}}{j !}\left(\begin{array}{c}
n-\frac{1}{2} \\
n-l-1-j
\end{array}\right)(4 B \lambda+4)^{-\frac{l+2 j+3}{2}} \\
& \times \Gamma(l+2 j+3) \sum_{i=0}^{\lfloor l / 2\rfloor} \frac{(-1)^{l-i}(2 l-2 i) !\left(1+(-1)^{l-2 i}\right)}{2^{l} i !(l-i) !(l-2 i) !} \times \Phi_{l, j, i}\left(p_{\lambda, r_{0}}\right) .
\end{aligned}
$$

and $\widehat{g}_{n l m}^{\lambda}=0$, if $m \neq 0$.

Proof. The function $g\left(\boldsymbol{x}-\boldsymbol{x}_{0}\right)=\exp \left(-B\left|\boldsymbol{x}-\boldsymbol{x}_{0}\right|^{2}\right)$ can be rewritten in the spherical coordinates $\boldsymbol{x}=(r, \theta, \varphi)$ in the following way

$$
g(r, \theta)=\exp \left(-B\left(r_{0}^{2}+r^{2}-2 r r_{0} \cos \theta\right)\right) .
$$

Since $\overline{Y_{l, m}(\theta, \varphi)}=(-1)^{m} Y_{l,-m}(\theta, \varphi)$, we have

$$
\begin{aligned}
\widehat{g}_{n l m}^{\lambda}= & \int_{0}^{\pi} \int_{0}^{2 \pi} \int_{0}^{\infty} g(r, \theta) \overline{H_{n l m}^{\lambda}(r, \theta, \varphi)} \exp \left(-r^{2} / \lambda\right) r^{2} \sin \theta \mathrm{d} r \mathrm{~d} \varphi \mathrm{d} \theta \\
= & {\left[\frac{2(n-l-1) !}{\lambda^{\frac{3}{2}} \Gamma\left(n+\frac{1}{2}\right)}\right]^{\frac{1}{2}}\left[\frac{(2 l+1)(l+m) !}{4 \pi(l-m) !}\right]^{\frac{1}{2}}(-1)^{m} \int_{0}^{2 \pi} \exp (-\mathrm{i} m \varphi) \mathrm{d} \varphi } \\
& \times \int_{0}^{\pi} \int_{0}^{\infty} g(r, \theta) \exp \left(-r^{2} / \lambda\right)(r / \sqrt{\lambda})^{l} L_{n-l-1}^{l+\frac{1}{2}}\left(r^{2} / \lambda\right) P_{l,-m}(\cos \theta) r^{2} \sin \theta \mathrm{d} r \mathrm{~d} \theta .
\end{aligned}
$$

Taking into account that

$$
\int_{0}^{2 \pi} \exp (-\mathrm{i} m \varphi) \mathrm{d} \varphi= \begin{cases}2 \pi, & m=0 \\ 0, & m \neq 0\end{cases}
$$

we obtain $\widehat{g}_{n l m}^{\lambda}=0$ if $m \neq 0$.

Let now $m=0$. For simplicity we let

$$
\varpi_{n l}^{\lambda}:=\exp \left(B r_{0}^{2}\right)\left[\frac{2 \pi \lambda^{\frac{3}{2}}(n-l-1) !(2 l+1)}{\Gamma\left(n+\frac{1}{2}\right)}\right]^{-\frac{1}{2}} \widehat{g}_{n l 0}^{\lambda} .
$$

Making the change of variables $r / \sqrt{\lambda} \rightarrow t$ in (4.2), we obtain

$$
\varpi_{n l}^{\lambda}=\int_{0}^{\pi} \int_{0}^{\infty} \exp \left(2 B \sqrt{\lambda} t r_{0} \cos \theta\right) \exp \left(-(B \lambda+1) t^{2}\right) t^{l} L_{n-l-1}^{l+\frac{1}{2}}\left(t^{2}\right) P_{l}(\cos \theta) t^{2} \sin \theta \mathrm{d} t \mathrm{~d} \theta .
$$

Using the closed form for the associated Laguerre polynomials [7, 8.970 (1)]

$$
L_{n}^{\alpha}(x)=\sum_{j=0}^{n}(-1)^{j}\left(\begin{array}{c}
n+\alpha \\
n-j
\end{array}\right) \frac{x^{j}}{j !}
$$


we get

$$
\begin{aligned}
\varpi_{n l}^{\lambda}= & \sum_{j=0}^{n-l-1} \frac{(-1)^{j}}{j !}\left(\begin{array}{c}
n-\frac{1}{2} \\
n-l-1-j
\end{array}\right) \\
& \times \int_{0}^{\pi} \int_{0}^{\infty} t^{(l+2 j+3)-1} \exp \left(2 B \sqrt{\lambda} t r_{0} \cos \theta\right) \exp \left(-(B \lambda+1) t^{2}\right) P_{l}(\cos \theta) \sin \theta \mathrm{d} t \mathrm{~d} \theta .
\end{aligned}
$$

From the formula [7, 3.462 (1)] for $\beta, \nu>0$

$$
\int_{0}^{\infty} x^{\nu-1} \exp \left(-\beta x^{2}-\gamma x\right) \mathrm{d} x=(2 \beta)^{-\frac{\nu}{2}} \Gamma(\nu) \exp \left(\frac{\gamma^{2}}{8 \beta}\right) \mathrm{D}_{-\nu}\left(\frac{\gamma}{\sqrt{2 \beta}}\right),
$$

where $\mathrm{D}$ is the parabolic cylinder function (see [7, 9.24-9.25]), we have

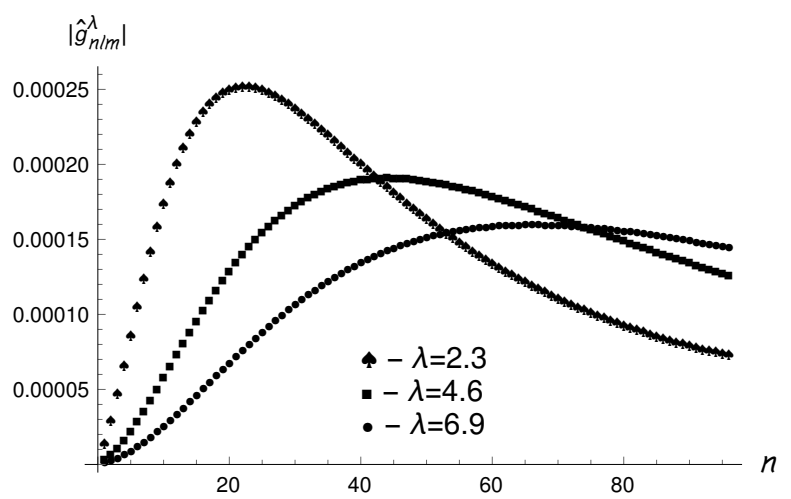
$m=3$ ).

FIG. 4.1. Graphic of the behavior of $\left|\widehat{g}_{n l m}^{\lambda}\right|\left(B=2, r_{0}=1, \theta_{0}=\frac{\pi}{6}, \varphi_{0}=\frac{\pi}{3}\right)$ with respect to $n(l=4$,

$$
\begin{aligned}
\varpi_{n l}^{\lambda}= & \sum_{j=0}^{n-l-1} \frac{(-1)^{j}}{j !}\left(\begin{array}{c}
n-\frac{1}{2} \\
n-l-1-j
\end{array}\right)(2 B \lambda+2)^{-\frac{l+2 j+3}{2}} \Gamma(l+2 j+3) \\
& \times \int_{0}^{\pi} \exp \left(\frac{1}{4} p_{\lambda, r_{0}}^{2} \cos ^{2} \theta\right) \mathrm{D}_{-l-2 j-3}\left(-p_{\lambda, r_{0}} \cos \theta\right) P_{l}(\cos \theta) \sin \theta \mathrm{d} \theta
\end{aligned}
$$

where $p_{\lambda, r_{0}}=\frac{2 B \sqrt{\lambda} r_{0}}{\sqrt{2 B \lambda+2}}$.

Let us define

$$
\begin{aligned}
I: & =\int_{0}^{\pi} \exp \left(\frac{1}{4} p_{\lambda, r_{0}}^{2} \cos ^{2} \theta\right) \mathrm{D}_{-l-2 j-3}\left(-p_{\lambda, r_{0}} \cos \theta\right) P_{l}(\cos \theta) \sin \theta \mathrm{d} \theta \\
& =\int_{-1}^{1} \exp \left(\frac{1}{4} p_{\lambda, r_{0}}^{2} t^{2}\right) \mathrm{D}_{-l-2 j-3}\left(-p_{\lambda, r_{0}} t\right) \mathrm{P}_{l}(t) \mathrm{d} t .
\end{aligned}
$$


By using the monomial representation of the Legendre polynomial [7, 8.911 (1)]

$$
P_{l}(t)=\sum_{i=0}^{\lfloor l / 2\rfloor}(-1)^{i} \frac{(2 l-2 i) !}{2^{l} i !(l-i) !(l-2 i) !} t^{l-2 i},
$$

we have

$$
I=\sum_{i=0}^{\lfloor l / 2\rfloor}(-1)^{i} \frac{(2 l-2 i) !}{2^{l} i !(l-i) !(l-2 i) !} \int_{-1}^{1} \exp \left(\frac{1}{4} p_{\lambda, r_{0}}^{2} t^{2}\right) \mathrm{D}_{-l-2 j-3}\left(-p_{\lambda, r_{0}} t\right) t^{l-2 i} \mathrm{~d} t .
$$

Making a change of variables $-p_{\lambda, r_{0}} t \rightarrow y$, we obtain

$$
I=\sum_{i=0}^{\lfloor l / 2\rfloor} \frac{(-1)^{i+1}(2 l-2 i) !}{2^{l} i !(l-i) !(l-2 i) !}\left(-\frac{1}{p_{\lambda, r_{0}}}\right)^{l-2 i+1} \int_{-p_{\lambda, r_{0}}}^{p_{\lambda, r_{0}}} y^{l-2 i} \exp \left(y^{2} / 4\right) \mathrm{D}_{-l-2 j-3}(y) \mathrm{d} y .
$$

From the formula ${ }^{1}$

$$
\begin{aligned}
\int z^{\mu-1} \exp \left(z^{2} / 4\right) \mathrm{D}_{\nu}(z) \mathrm{d} z= & \frac{\sqrt{\pi} 2^{\frac{\nu}{2}} z^{\mu}}{\mu \Gamma\left(\frac{1-\nu}{2}\right)}{ }_{2} F_{2}\left(\frac{\mu}{2},-\frac{\nu}{2} ; \frac{1}{2}, \frac{\mu}{2}+1 ; \frac{z^{2}}{2}\right) \\
& -\frac{\sqrt{\pi} 2^{\frac{\nu+1}{2}} z^{\mu+1}}{(\mu+1) \Gamma\left(\frac{-\nu}{2}\right)}{ }_{2} F_{2}\left(\frac{\mu+1}{2}, \frac{1-\nu}{2} ; \frac{3}{2}, \frac{\mu+3}{2} ; \frac{z^{2}}{2}\right)
\end{aligned}
$$

we obtain

$$
I=\sqrt{\pi} \sum_{i=0}^{\lfloor l / 2\rfloor}(-1)^{l-i} \frac{(2 l-2 i) !\left(1+(-1)^{l-2 i}\right)}{2^{l} i !(l-i) !(l-2 i) !} 2^{-\frac{l+2 j+3}{2}} \times \Phi_{l, j, i}\left(p_{\lambda, r_{0}}\right) .
$$

Relations (4.3), (4.4) and (4.5), taking into account definition of $\varpi_{n l}^{\lambda}$, imply (4.1).

II. Before formulating our next results let us first describe the main ideas of the rotational invariance property of spherical harmonics. We consider the expansion of a function $f \in$ $L_{2}\left(\mathbb{R}^{3}, \omega_{\lambda}\right)$ in the SGL Fourier series. Taking into account the definition of the set $\nabla_{n}$ we obtain

$$
f(r, \theta, \varphi)=\sum_{n=1}^{\infty} \sum_{l=0}^{n-1} \sum_{m=-l}^{l} \widehat{f}_{n l m}^{\lambda} H_{n l m}^{\lambda}(r, \theta, \varphi)=\sum_{n=1}^{\infty} \sum_{l=0}^{n-1} \sum_{m=-l}^{l} \widehat{f}_{n l m}^{\lambda} R_{n l}^{\lambda}(r) Y_{l m}(\theta, \varphi),
$$

where the convergence is understood in the sense of the space $L_{2}\left(\mathbb{R}^{3}, \omega_{\lambda}\right)$.

Applying to the function $f$ the Euler rotation operator $\widehat{R}(\alpha, \beta, \gamma), \alpha, \gamma \in[0,2 \pi)$ and $\beta \in[0, \pi]$, we get (see, for example, [15, p. 37-44] for detailed information on the Euler rotation operator and the transformations below)

$$
\begin{aligned}
\widehat{R}(\alpha, \beta, \gamma) f(r, \theta, \varphi) & =\sum_{n=1}^{\infty} \sum_{l=0}^{n-1} \sum_{m=-l}^{l} \widehat{f}_{n l m}^{\lambda} R_{n l}^{\lambda}(r) \widehat{R}(\alpha, \beta, \gamma) Y_{l m}(\theta, \varphi) \\
& =\sum_{n=1}^{\infty} \sum_{l=0}^{n-1} \sum_{m=-l}^{l} \widehat{f}_{n l m}^{\lambda} R_{n l}^{\lambda}(r) \sum_{m^{\prime}=-l}^{l} Y_{l m^{\prime}}(\theta, \varphi) \mathbf{D}_{m^{\prime} m}^{(l)}(\alpha, \beta, \gamma) \\
& =\sum_{n=1}^{\infty} \sum_{l=0}^{n-1} \sum_{m^{\prime}=-l}^{l}\left(\sum_{m=-l}^{l} \mathbf{D}_{m^{\prime} m}^{(l)}(\alpha, \beta, \gamma) \widehat{f}_{n l m}^{\lambda}\right) R_{n l}^{\lambda}(r) Y_{l m^{\prime}}(\theta, \varphi)
\end{aligned}
$$

\footnotetext{
${ }^{1}$ Wolfram website: http://functions.wolfram.com/HypergeometricFunctions/ ParabolicCylinderD/21/01/01/02/0001/
} 
where $\mathbf{D}_{m^{\prime} m}^{(l)}$ are the Wigner rotation matrices defined as follows

$$
\mathbf{D}_{m^{\prime} m}^{(l)}(\alpha, \beta, \gamma)=\exp \left(-\mathrm{i} m^{\prime} \alpha\right) d_{m^{\prime} m}^{(l)}(\beta) \exp (-\mathrm{i} m \gamma)
$$

and

$$
\begin{aligned}
d_{m^{\prime} m}^{(l)}(\beta)= & {\left[\left(l+m^{\prime}\right) !\left(l-m^{\prime}\right) !(l+m) !(l-m) !\right]^{\frac{1}{2}} } \\
& \times \sum_{k=\max \left\{0, m-m^{\prime}\right\}}^{\min \left\{l-m^{\prime}, l+m\right\}} \frac{(-1)^{k+m^{\prime}-m}\left[\cos \left(\frac{\beta}{2}\right)\right]^{2 l+m-m^{\prime}-2 k}\left[\sin \left(\frac{\beta}{2}\right)\right]^{2 k+m^{\prime}-m}}{(l+m-k) ! k !\left(m^{\prime}-m+k\right) !\left(l-m^{\prime}-k\right) !} .
\end{aligned}
$$

In order words, if $\widehat{f}_{n l m}^{\lambda}$ are the SGL Fourier coefficients of a function $f$, the SGL Fourier coefficients of the function $h=\widehat{R}(\alpha, \beta, \gamma) f$ can be found by the formula

$$
\widehat{h}_{n l m}^{\lambda}=\sum_{m^{\prime}=-l}^{l} \mathbf{D}_{m m^{\prime}}^{(l)}(\alpha, \beta, \gamma) \widehat{f}_{n l m^{\prime}}^{\lambda}
$$

Finally, using the relation (4.6) and Theorem 4.1 we obtain formulas for the SGL Fourier coefficients of the function $g\left(\boldsymbol{x}-\boldsymbol{x}_{0}\right)=\exp \left(-B\left|\boldsymbol{x}-\boldsymbol{x}_{0}\right|^{2}\right)$, where in the spherical coordinates $\boldsymbol{x}_{0}=\left(r_{0}, \theta_{0}, \varphi_{0}\right)$, and

$$
g(r, \theta, \varphi)=\exp \left(-B r_{0}^{2}-B r^{2}+2 B r r_{0}\left(\sin \theta \sin \theta_{0} \cos \left(\varphi-\varphi_{0}\right)+\cos \theta \cos \theta_{0}\right)\right) .
$$

Corollary 4.2. For the function $g\left(\boldsymbol{x}-\boldsymbol{x}_{0}\right)=\exp \left(-B\left|\boldsymbol{x}-\boldsymbol{x}_{0}\right|^{2}\right), B>0, \boldsymbol{x}, \boldsymbol{x}_{0} \in \mathbb{R}^{3}$, $\boldsymbol{x}_{0}=\left(r_{0}, \theta_{0}, \varphi_{0}\right)$ and $\lambda>0$, the following formulas hold

$$
\widehat{g}_{n l m}^{\lambda}=\mathbf{D}_{m 0}^{(l)}\left(\varphi_{0}, \theta_{0}, 0\right) \widehat{g}_{n l 0}^{\lambda},
$$

where $\widehat{g}_{n l 0}^{\lambda}$ is defined by (4.1).

The behavior of $\left|\widehat{g}_{n l m}^{\lambda}\right|$ with respect to the indices $n, l$, and $m$ with fixed values for the other indices is illustrated in Figures 4.1 and 4.2.
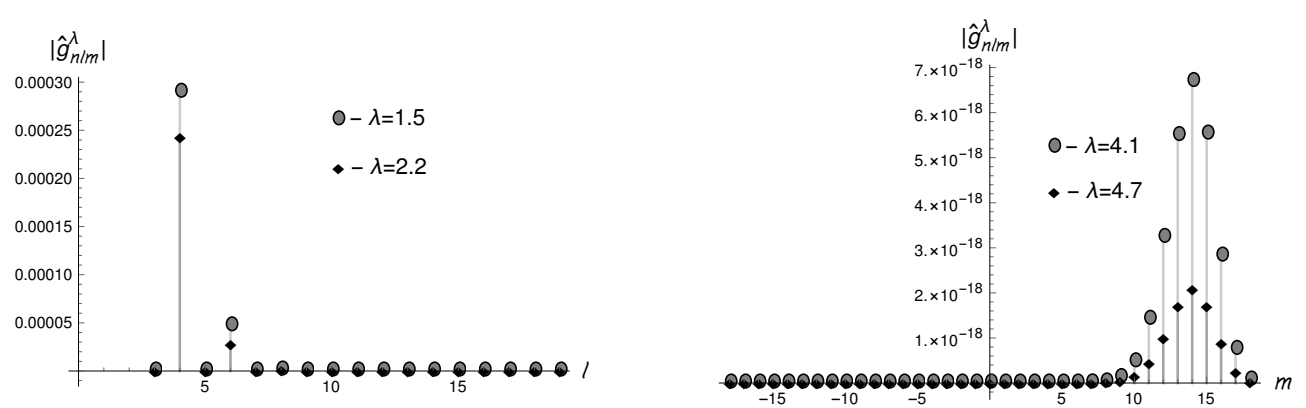

FIG. 4.2. Graphics of the behavior of $\left|\widehat{g}_{n l m}^{\lambda}\right|\left(B=2, r_{0}=1, \theta_{0}=\frac{\pi}{6}, \varphi_{0}=\frac{\pi}{3}\right.$ ) with respect to l (left, $n=20, m=3$ ) and with respect to $m$ (right, $n=20, l=18$ ).

For the case when $\boldsymbol{x}_{0} \neq(0,0,0)$ it seems to be impossible to write results similar to Theorems 3.2, 3.4, and 3.5 due to complex analytic representation of the SGL Fourier coefficients of Gaussians (see Theorem 4.1 and Corollary 4.2). Therefore, in this case we use a computational method to investigate the behavior of the quantity $\mathcal{E}_{N}\left(g, \mathcal{H}^{\lambda}\right)$. Let us describe the main ideas of this method. 
By Parseval's equality, we have

$$
\begin{aligned}
\mathcal{E}_{N}\left(g, \mathcal{H}^{\lambda}\right) & =\left\|g-\sum_{n=1}^{N}\left(\sum_{(l, m) \in \nabla n} \widehat{g}_{n l m}^{\lambda} H_{n l m}^{\lambda}\right)\right\|_{L_{2}\left(\mathbb{R}^{3}, \omega_{\lambda}\right)} \\
& =\left(\sum_{n=N+1}^{\infty}\left(\sum_{(l, m) \in \nabla n}\left|\widehat{g}_{n l m}^{\lambda}\right|^{2}\right)\right)^{\frac{1}{2}} .
\end{aligned}
$$

Let us choose $N_{0} \in \mathbb{N}$ from the condition

$$
\sum_{(l, m) \in \nabla n}\left|\widehat{g}_{n l m}^{\lambda}\right|^{2}<\varepsilon \text { for } n>N_{0},
$$

where $\varepsilon$ is a very small positive number. Then we can assume that

$$
\mathcal{E}_{N}\left(g, \mathcal{H}^{\lambda}\right) \approx\left(\sum_{n=N+1}^{N_{0}}\left(\sum_{(l, m) \in \nabla n}\left|\widehat{g}_{n l m}^{\lambda}\right|^{2}\right)\right)^{\frac{1}{2}} .
$$

By using (4.7) and the formulas for the SGL Fourier coefficients $\widehat{g}_{n l m}^{\lambda}$ from the Corollary 4.2, we can numerically compute approximate values of $\mathcal{E}_{N}\left(g, \mathcal{H}^{\lambda}\right)$. Analyzing numerical data we can conclude that in case when $g\left(\boldsymbol{x}-\boldsymbol{x}_{0}\right)=\exp \left(-B\left|\boldsymbol{x}-\boldsymbol{x}_{0}\right|^{2}\right)$ and $\boldsymbol{x}_{0} \neq 0$ the quantity $\mathcal{E}_{N}\left(g, \mathcal{H}^{\lambda}\right)$ keeps its behavior with respect to the parameter $\lambda$ (compare Figure 3.1 (right) and Figure 4.3 (right)).
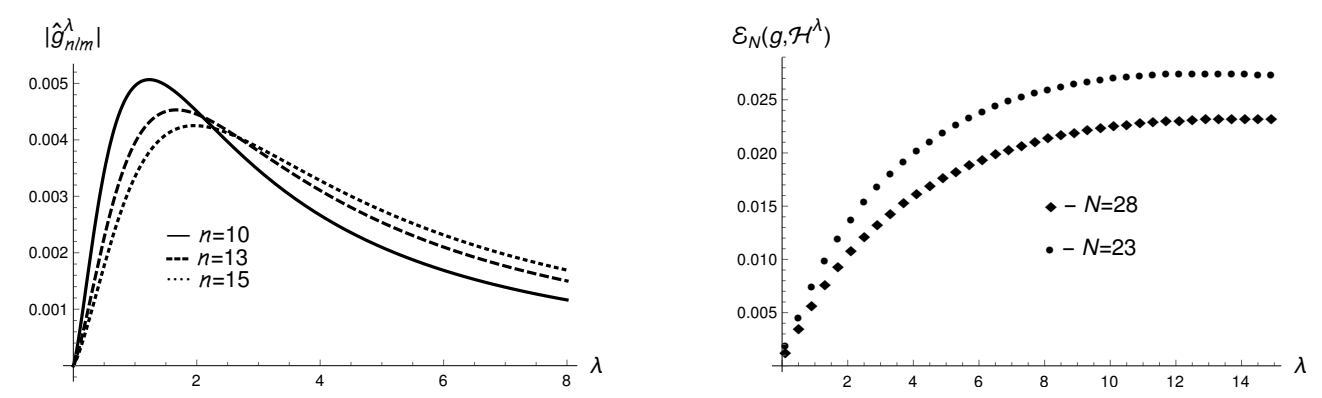

FIG. 4.3. Graphics of the behavior of $\left|\widehat{g}_{n l m}^{\lambda}\right|$ (left, $B=2, \boldsymbol{x}_{0}=(1,0,0)$ in the spherical coordinates, $l=2$, $m=0)$ and $\mathcal{E}_{N}\left(g, \mathcal{H}^{\lambda}\right)$ (right, $B=2$ and $\boldsymbol{x}_{0}=(1,0,0)$ in the spherical coordinates) with respect to $\lambda$.

5. Discussion, conclusions, and possible applications. In this section we describe one possible application of the obtained results to the problem of molecular docking. Let us start with some known facts.

Gaussian functions are often used to approximate the electron density distribution of molecules ([8], [9]). For example, for the $k$ th atom we have

$$
\mathcal{K}\left(\boldsymbol{x}-\boldsymbol{x}_{c k}\right)=\exp \left(\frac{B\left|\boldsymbol{x}-\boldsymbol{x}_{k}\right|^{2}}{r_{k}^{2}}-B\right)
$$

where $B<0$ is the rate of decay parameter, $r_{k}$ is the Van der Waals radius of the $k$ th atom and $\left|\boldsymbol{x}-\boldsymbol{x}_{k}\right|^{2}=\left(x-x_{k}\right)^{2}+\left(y-y_{k}\right)^{2}+\left(z-z_{k}\right)^{2}$, where $\boldsymbol{x}_{k}=\left(x_{k}, y_{k}, z_{k}\right)$ is the center of the 
$k$ th atom. A volumetric representation of the molecule may now be obtained by summing the contributions from each single atom, i.e., the electron density for a molecule with $M$ atoms is described as

$$
\sum_{k=1}^{M} \mathcal{K}\left(\boldsymbol{x}-\boldsymbol{x}_{k}\right)=\sum_{k=1}^{M} \exp \left(\frac{B\left|\boldsymbol{x}-\boldsymbol{x}_{k}\right|^{2}}{r_{k}^{2}}-B\right) .
$$

The choice of a suitable scoring function is the crucial part in all docking approaches. Some papers (see, for instance, [11]) consider the approach of separating the affinity functions into core and skin regions with the further goal to penalize core-core clashes and add positive skin-skin overlaps. By using positive real values as the weights for the smooth particle representation of the affinity function defined over the skin and imaginary values in the representation of the core regions, we will obtain negative numbers for core-core overlaps and positive numbers for skin-skin overlaps during the convolution. So, the weighted affinity function for the molecule $\mathbf{A}$ takes the form

$$
Q^{\mathbf{A}}(\boldsymbol{x})=\sum_{k=1}^{M} \gamma_{k}^{\mathbf{A}} \mathcal{K}\left(\boldsymbol{x}-\boldsymbol{x}_{k}\right)
$$

where

$$
\gamma_{k}^{\mathbf{A}}= \begin{cases}1, & \boldsymbol{x}_{k} \in \operatorname{Skin} \mathbf{A} \\ \rho \mathrm{i}, & \boldsymbol{x}_{k} \in \operatorname{Core} \mathbf{A}\end{cases}
$$

and $\rho \gg 1$.

Let $\widehat{R}(\alpha, \beta, \gamma)$ and $\widehat{R}^{\prime}\left(0, \beta^{\prime}, \gamma^{\prime}\right)$ be the Euler rotation operators (see Section 4 and [15, p. 37-44]). The notation $Q_{\widehat{R}}^{\mathbf{A}}$ means that we apply the operator $\widehat{R}$ to the function $Q^{\mathbf{A}}$. By $\mathrm{T}^{\tau}$ we denote the operator of the shift on the vector $\boldsymbol{\tau}=(\tau, 0,0)$, i.e., $\mathrm{T}^{\boldsymbol{\tau}} Q^{\mathbf{A}}(\boldsymbol{x})=Q^{\mathbf{A}}(\boldsymbol{x}-\boldsymbol{\tau})$. We consider the search algorithm suggested by Ritchie [15, Chapter 4]. The main ideas of this algorithm is to use a Fast Rotational Matching approach, i.e., considering of the $6 D$ search space $\left(\alpha, \beta, \gamma, \beta^{\prime}, \gamma^{\prime}, \tau\right)$, where two molecules $\mathbf{A}$ and $\mathbf{B}$ to be docked are understood to be flexible during the docking.

By $\operatorname{Re} c$ we denote the real part of the complex number $c \in \mathbb{C}$. Then the convolution search scoring is defined by

$$
C\left(\widehat{R}, \widehat{R}^{\prime}, \boldsymbol{t}\right)=\operatorname{Re}\left(\int_{\mathbb{R}^{3}} Q_{\widehat{R}}^{\mathbf{A}}(\boldsymbol{x}) \mathrm{T}^{\boldsymbol{\tau}} Q_{\widehat{R}^{\prime}}^{\mathbf{B}}(\boldsymbol{x}) \omega_{\lambda}(\boldsymbol{x}) \mathrm{d} \boldsymbol{x}\right)
$$

and the solution of the docking problem is the pair $\left(\widehat{R}_{\max }, \widehat{R}_{\max }^{\prime}, \boldsymbol{\tau}_{\max }\right)$ for which

$$
C\left(\widehat{R}_{\max }, \widehat{R}_{\max }^{\prime}, \boldsymbol{\tau}_{\max }\right)=\max _{\left(\widehat{R}, \widehat{R}^{\prime}, \boldsymbol{\tau}\right)} C\left(\widehat{R}, \widehat{R}^{\prime}, \boldsymbol{\tau}\right) .
$$

By using expansions of the corresponding scoring functions in the SGL Fourier series we get

$$
C\left(\widehat{R}, \widehat{R}^{\prime}, \boldsymbol{\tau}\right) \approx \operatorname{Re}\left(\sum_{n l m}^{N} \sum_{n^{\prime} l^{\prime} m^{\prime}}^{N} \int_{\mathbb{R}^{3}}\left(\widehat{\left(Q_{\widehat{R}}^{\mathbf{A}}\right)_{n l m}^{\lambda}} \widehat{\left(\mathrm{T}^{\boldsymbol{\tau}} Q_{\widehat{R}^{\prime}}^{\mathrm{B}}\right.}\right)_{n l m}^{\lambda} H_{n l m}^{\lambda}(\boldsymbol{x}) H_{n^{\prime} l^{\prime} m^{\prime}}^{\lambda}(\boldsymbol{x}) \omega_{\lambda}(\boldsymbol{x}) \mathrm{d} \boldsymbol{x}\right) .
$$

The docking integral (5.1) depends on the scale factor $\lambda$. For the optimization of the computations of scoring for given molecules $\mathbf{A}$ and $\mathbf{B}$ one should use a suitable value for the 
parameter $\lambda$. In [15, p. 34] it is shown that with the order of expansion $N=28$, the SGL basis functions give good level of recovery of the molecular shape. To translate and scale the docking region to the unit ball $\mathbb{S}^{2}$ and to be oriented on the most distant atom one can choose values of $\lambda$ based on Figure 4.3 (right). However, values really close to zero, probably, cannot be used since then the weighted Gaussian function together with basis functions tend to zero and the basis elements cannot provide good sampling for the recovery of the molecular shape. On the other hand, using a small $\lambda$ in naive computations can cause computational inaccuracy.

Acknowledgments. The authors were supported by H2020-MSCA-RISE-2014 Project number 645672 (AMMODIT: Approximation Methods for Molecular Modelling and Diagnosis Tools). The authors would like to thank the referees for their valuable comments regarding the paper. Moreover, the authors would like to thank Viktor Romanyuk (Institute of Mathematics NAS Ukraine) for many helpful scientific discussions.

\section{REFERENCES}

[1] M. Abramowitz and I. A. Stegun, Handbook of Mathematical Functions with Formulas, Graphs, and Mathematical Tables, vol. 55 of National Bureau of Standards Applied Mathematics Series, U.S. Government Printing Office, Washington, 1964.

[2] G. E. ANDrews, R. AsKey, AND R. RoY, Special Functions, vol. 71 of Encyclopedia of Mathematics and its Applications, Cambridge University Press, Cambridge, 1999.

[3] K. AtKinson AND W. Han, Spherical Harmonics and Approximations on the Unit Sphere: An Introduction, Springer, Heidelberg, 2012.

[4] F. Dai AND Y. Xu, Approximation Theory and Harmonic Analysis on Spheres and Balls, Springer, New York, 2013.

[5] C. F. DunkL And Y. Xu, Orthogonal Polynomials of Several Variables, 2nd ed., vol. 155 of Encyclopedia of Mathematics and its Applications, Cambridge University Press, Cambridge, 2014.

[6] N. Elezović, Asymptotic expansions of gamma and related functions, binomial coefficients, inequalities and means, J. Math. Inequal., 9 (2015), pp. 1001-1054.

[7] I. S. GRadshteyn AND I. M. RyzhiK, Table of Integrals, Series, and Products, Academic Press, Boston, 1994.

[8] J. A. GRANT, M. A. Gallardo, AND B. T. Pickup, A fast method of molecular shape comparison: A simple application of a Gaussian description of molecular shape, J. Comp. Chem., 17 (1996), pp. 1653-1666.

[9] J. A. GRAnT AND B. T. PickUP, A Gaussian description of molecular shape, J. Phys. Chem, 99 (1995), pp. 3503-3510.

[10] A. I. KAMzoLOV, The best approximation of classes of functions $W_{p}^{\alpha}\left(S^{n}\right)$ by polynomials in spherical harmonics, Mat. Zametki, 32 (1982), pp. 285-293, 425.

[11] E. Katchalski-Katzir, I. Shariv, M. Eisenstein, A. A. Friesem, C. Aflalo, and I. A. Vakser, Molecular surface recognition: determination of geometric fit between proteins and their ligands by correlation techniques., Proc. Natl. Acad. Sci. USA, 89 (1992), pp. 2195-2199.

[12] L. MAK, S. GRANDISON, AND R. J. MORRIS, An extension of spherical harmonics to region-based rotationally invariant descriptors for molecular shape description and comparison, J. Molecular Graph. Model., 26 (2008), pp. 1035-1045.

[13] M. Novotni AND R. KLEIN, Shape retrieval using 3D Zernike descriptors, Computer-Aided Design, 36 (2004), pp. 1047-1062.

[14] J. PRESTIN AND C. WÜLKER, Fast Fourier transforms for spherical Gauss-Laguerre basis functions, in Frames and Other Bases in Abstract and Function Spaces, I. Pesenson, Q. Thong Le Gia, A. Mayeli, H. Mhaskar, and Ding-Xuan Zhou, eds., Appl. Numer. Harmon. Anal., Birkhäuser, Cham, 2017, pp. $237-$ 263.

[15] D. RitchiE, High Performance Algorithms for Molecular Shape Recognition, Habilitation Memoir, CNU Section 27-Informatique, 2011.

[16] D. Ritchie AND G. Kemp, Protein docking using spherical polar Fourier correlations, Proteins, 39 (2000), pp. 178-94.

[17] V. S. RomANyUK, Nonlinear widths of classes of smooth functions defined on the unit sphere in $\mathbb{R}^{d}$, Mat. Zametki, 85 (2009), pp. 147-152.

[18] D. WANG, S. SUn, X. ChEn, AND Z. YU, A 3D shape descriptor based on spherical harmonics through evolutionary optimization, Neurocomputing, 194 (2016), pp. 183-191. 